Asymptotic expansions and fast computation of oscillatory Hilbert transforms

Haiyong Wang, Lun Zhang and Daan Huybrechs

Report TW605, December 2011

Katholieke Universiteit Leuven
Department of Computer Science

Celestijnenlaan 200A - B-3001 Heverlee (Belgium) 


\title{
Asymptotic expansions and fast computation of oscillatory Hilbert transforms
}

\author{
Haiyong Wang, Lun Zhang and Daan Huybrechs \\ Report TW605, December 2011
}

Department of Computer Science, K.U.Leuven

\begin{abstract}
In this paper, we study the asymptotics and fast computation of the one-sided oscillatory Hilbert transforms of the form

$$
H^{+}\left(f(t) e^{i \omega t}\right)(x)=f_{0}^{\infty} e^{i \omega t} \frac{f(t)}{t-x} d t, \quad \omega>0, \quad x \geq 0,
$$

where the bar indicates the Cauchy principal value and $f$ is a realvalued function with analytic continuation in the first quadrant, except possibly a branch point of algebraic type at the origin. When $x=0$, the integral is interpreted as a Hadamard finite-part integral, provided it is divergent. Asymptotic expansions in inverse powers of $\omega$ are derived for each fixed $x \geq 0$, which clarify the large $\omega$ behavior of this transform. We then present efficient and affordable approaches for numerical evaluation of such oscillatory transforms. Depending on the position of $x$, we classify our discussion into three regimes, namely, $x=\mathcal{O}(\infty)$ or $x \gg 1,0<x \ll 1$ and $x=0$. Numerical experiments show that the convergence of the proposed methods greatly improve when the frequency $\omega$ increases. Some extensions to oscillatory Hilbert transforms with Bessel oscillators are briefly discussed as well.
\end{abstract}

Keywords : Cauchy principal value, high oscillation, asymptotic expansions, numerical steepest descent methods

AMS(MOS) Classification : Primary : 34E05, Secondary : 65D32, 44A15. 


\title{
Asymptotic expansions and fast computation of oscillatory Hilbert transforms
}

\author{
Haiyong Wang*t Lun Zhang ${ }^{\ddagger}$ and Daan Huybrechs* ${ }^{* \dagger}$
}

\begin{abstract}
In this paper, we study the asymptotics and fast computation of the one-sided oscillatory Hilbert transforms of the form

$$
H^{+}\left(f(t) e^{i \omega t}\right)(x)=f_{0}^{\infty} e^{i \omega t} \frac{f(t)}{t-x} d t, \quad \omega>0, \quad x \geq 0,
$$

where the bar indicates the Cauchy principal value and $f$ is a real-valued function with analytic continuation in the first quadrant, except possibly a branch point of algebraic type at the origin. When $x=0$, the integral is interpreted as a Hadamard finite-part integral, provided it is divergent. Asymptotic expansions in inverse powers of $\omega$ are derived for each fixed $x \geq 0$, which clarify the large $\omega$ behavior of this transform. We then present efficient and affordable approaches for numerical evaluation of such oscillatory transforms. Depending on the position of $x$, we classify our discussion into three regimes, namely, $x=\mathcal{O}(1)$ or $x \gg 1,0<x \ll 1$ and $x=0$. Numerical experiments show that the convergence of the proposed methods greatly improve when the frequency $\omega$ increases. Some extensions to oscillatory Hilbert transforms with Bessel oscillators are briefly discussed as well.
\end{abstract}

Keywords: Cauchy principal value, high oscillation, asymptotic expansions, numerical steepest descent methods.

AMS classifications: 34E05, 65D32, 44A15.

\section{Introduction}

Finite Fourier integrals of the form

$$
\int_{a}^{b} f(x) e^{i \omega g(x)} d x
$$

${ }^{*}$ Department of Computer Science, Katholieke Universiteit Leuven, Celestijnenlaan 200A, B-3001 Leuven, Belgium (why198309@yahoo.com.cn, haiyong.wang@cs.kuleuven.be., daan.huybrechs@cs.kuleuven.be)

${ }^{\dagger}$ This research was supported by the Fund for Scientific Research - Flanders through Research Project G.0617.10.

${ }^{\ddagger}$ Department of Mathematics, Katholieke Universiteit Leuven, Celestijnenlaan 200B, BE-3001 Leuven, Belgium (lun.zhang@wis.kuleuven.be). This author is a Postdoctoral Fellow of the Fund for Scientific Research - Flanders (FWO), Belgium. 
with $\omega>0$ and $f(x), g(x)$ being sufficiently smooth functions have long been the subject of intensive study due to their frequent occurrences in wide fields ranging from quantum chemistry, image analysis, electrodynamics and computerized tomography to fluid mechanics [15. One difficulty in computing integrals (1.1) is that, for large frequency $\omega$, the classical integration methods like Gauss quadrature are inapplicable, since they often require many function evaluations which make them highly time consuming. To overcome this difficulty, many efficient approaches have been developed and significant progress has occurred over the past few years. For instance, based on asymptotic expansions of (1.1) as $\omega$ tends to infinity, Iserles and Nørsett [15, 16] proposed the asymptotic and Filon-type methods to evaluate oscillatory integrals numerically. Other efficient approaches include Levin-type methods, numerical steepest descent methods, generalized quadrature rules, GMRES methods, modified Clenshaw-Curtis methods, etc.; we refer to [3, 17, 8, 9, 14, 20, 21, 29, 30, 38, 39] and references therein for more information. All these methods complement each other but share the advantageous property that their accuracy improves greatly when $\omega$ increases.

Recently, oscillatory Hilbert transforms of the form

$$
f_{\Gamma} e^{i \omega t} \frac{f(t)}{t-x} d t, \quad x \in \Gamma
$$

have received considerable attention as well. Here, $\Gamma$ is an oriented curve in the complex plane, $f$ is a complex-valued function satisfying a Hölder condition, and the bar denotes Cauchy principal value. The interest in theoretical and numerical study of such integral transforms arises from the fact that many problems encountered in practice can be represented by an integral equation with an oscillatory kernel having a singularity of Cauchy type [5, 10, 18]; see also [17] for numerous applications of Hilbert transforms in applied sciences. Although the oscillatory Hilbert transforms (1.2) bear some resemblances with (1.1), nevertheless, the singularity of Cauchy type suggests special treatments. It will be especially interesting to see, as pointed out in [28], if the aforementioned methods can be extended to handle oscillatory Hilbert transforms.

For $\Gamma=[-1,1]$, we obtain from (1.2) the finite oscillatory Hilbert transforms:

$$
f_{-1}^{1} e^{i \omega t} \frac{f(t)}{t-x} d t=\lim _{\varepsilon \rightarrow 0^{+}}\left(\int_{-1}^{x-\varepsilon}+\int_{x+\varepsilon}^{1}\right) e^{i \omega t} \frac{f(t)}{t-x} d t
$$

with $-1<x<1$. An asymptotic expansion of (1.3) in inverse powers of $\omega$ was established by Lyness in 22 based on analytic continuation. Meanwhile, there are several numerical schemes available to calculate (1.3), most of which are typically based on interpolatory type techniques. For example, Okecha [26] proposed to compute (1.3) by using the Lagrange interpolation polynomial of degree $n$ interpolating $f$ at the $n+1$ zeros of the Legendre polynomial. Capobianco and Criscuolo introduced a numerically stable procedure in [5], which relies on an interpolatory procedure at the zeros of Jacobi polynomials. In a recent paper [33], Wang and Xiang have presented an integration rule of interpolatory type with the aid of the Chebyshev points of the second kind. The rule is uniformly convergent with respect to the pole $x$ when $f$ is analytic in a neighborhood 
of the interval $[-1,1]$, and it can be implemented by means of the fast Fourier transform (FFT). If the function $f$ is analytic in a sufficiently large region of the complex plane containing $[-1,1]$, then the complex integration method [23] and the numerical steepest descent method [14] can be extended to compute such integrals efficiently, and the accuracy improves greatly as $\omega$ increases; see [34 for details.

In this paper, we are concerned with one-sided oscillatory Hilbert transforms on the positive real axis:

$$
H^{+}\left(f(t) e^{i \omega t}\right)(x):=f_{0}^{\infty} e^{i \omega t} \frac{f(t)}{t-x} d t=\lim _{\varepsilon \rightarrow 0^{+}}\left(\int_{0}^{x-\varepsilon}+\int_{x+\varepsilon}^{\infty}\right) e^{i \omega t} \frac{f(t)}{t-x} d t, \quad x \geq 0,
$$

i.e., $\Gamma=\mathbb{R}^{+}$in (1.2). Here, $f$ is a real-valued function satisfying some conditions. In particular, it has an analytic continuation in the first quadrant of the complex plane, except possibly a branch point of algebraic type at the origin. When $x=0$, the integral is interpreted as a Hadamard finite-part integral, provided it is divergent; see Section 2.2 below for a definition. We point out that the integral (1.4) is also closely related to infinite oscillatory Hilbert transforms on the real axis given by

$$
H\left(f(t) e^{i \omega t}\right)(x):=f_{-\infty}^{\infty} e^{i \omega t} \frac{f(t)}{t-x} d t=\lim _{\varepsilon \rightarrow 0^{+}}\left(\int_{-\infty}^{x-\varepsilon}+\int_{x+\varepsilon}^{\infty}\right) e^{i \omega t} \frac{f(t)}{t-x} d t, \quad x \in \mathbb{R} .
$$

Indeed, by assuming $x>0$, it is easily seen that

$$
H\left(f(t) e^{i \omega t}\right)(x)=\int_{0}^{\infty}-e^{-i \omega t} \frac{f(-t)}{t+x} d t+H^{+}\left(f(t) e^{i \omega t}\right)(x)
$$

The first integral on the right hand side of (1.5) is the Stieltjes transform of $-e^{-i \omega t} f(-t)$, which is a regular integral for $x>0$.

A large $x$ expansion of one-sided oscillatory Hilbert transform was already established by Wong [35]. Instead of (1.4), he considered $H^{+}(f(t))(x)$. However, it is assumed that $f$ is a locally integrable function on $[0, \infty)$ and has an asymptotic expansion of the form

$$
f(t) \sim e^{i c t} \sum_{s=0}^{\infty} a_{s} t^{-s-\alpha}, \quad \text { as } \quad t \rightarrow \infty
$$

where $0<\alpha \leq 1$ and $c$ is a real number. Thus, one may have $c=\omega$. Let $\psi_{0}(t)=f(t)$ and define $\psi_{n}(t)$ by

$$
f(t)=\sum_{s=0}^{n-1} a_{s} e^{i c t} t^{-s-\alpha}+\psi_{n}(t), \quad n \geq 1 .
$$

It was then shown that (see [35, Thm. 1])

$$
H^{+}(f(t))(x)=E_{\alpha, c}(x) \sum_{s=0}^{n-1} \frac{a_{s}}{x^{s}}-\sum_{s=1}^{n} \frac{b_{s}}{x^{s}}+\frac{1}{x^{n}} \delta_{n}(x), \quad n \geq 1,
$$


for $0<\alpha<1$, where

$$
\begin{gathered}
E_{\alpha, c}(x)=\frac{e^{i c x}}{x^{\alpha}}\left[e^{-i \alpha \pi} \Gamma(1-\alpha) \Gamma(\alpha, i c x)+i \pi\right] \\
b_{s}=\int_{0}^{\infty} t^{s-1} \psi_{s}(t) d t, \quad s \geq 1
\end{gathered}
$$

and

$$
\delta_{n}(x)=f_{0}^{\infty} \frac{t^{n} \psi_{n}(t)}{t-x} d t, \quad n=0,1, \ldots
$$

Here $\Gamma(\alpha)$ is the Gamma function [2, p. 255] and $\Gamma(\alpha, z)$ is the complementary incomplete Gamma function [2, p. 260]. The expansions when $\alpha=1$ are also derived in a similar manner; see [35, Thm. 2 and Thm. 3]. Moreover, the following bounds for $\delta_{n}(x)$ are achieved:

$$
\left|\delta_{n}(x)\right| \leq M_{n} \frac{\ln x}{x^{\alpha}}
$$

for all $x>e$, where $M_{n}$ is a positive constant. The difficulty in applying expansions (1.6) is that, as also pointed out by Wong, the coefficients $b_{s}$ are inconvenient for calculations. In a later paper [32, Ursell generalized the results of Wong and showed that these coefficients can be readily determined whenever the Mellin transform of $f(t)$ is known. An interesting example was given for $f(t)=J_{0}^{2}(t)$ with applications in water waves, where $J_{0}(t)$ is the zeroth-order Bessel function of the first kind.

For the numerical aspects of (1.4), King et al. [18 constructed a fairly robust numerical procedure by using convergence accelerator techniques. Unfortunately, this series acceleration method may suffer from difficulties when the singularity is embedded in a region of extreme oscillatory behavior.

The purpose of this paper is two-fold. On the one hand, we shall derive asymptotic expansions of one-sided oscillatory Hilbert transforms (1.4) as $\omega \rightarrow \infty$. To the best of our knowledge, none of the studies are available in this direction. Such an expansion clarifies the behavior of (1.4) for large $\omega$ and also provides a powerful mean for the design of effective computational methods. On the other hand, in view of the fact that asymptotic expansions are not suitable for numerical calculation, we present efficient quadrature rules to approximate such integrals. It comes out that these rules depend on the position of $x$. This can be seen from (1.4) and (1.6), where the integral may tend to zero as $x \rightarrow \infty$ and blow up as $x \rightarrow 0$.

The rest of this paper is organized as follows. We perform asymptotic analysis of oscillatory Hilbert transforms in Section 2. The analyticity of $f$ is of importance in our derivation. In Section 3, we propose efficient and affordable approaches for numerical evaluation of such oscillatory transforms. These methods are designed for three regimes, that is, $x=\mathcal{O}(1)$ or $x \gg 1,0<x \ll 1$ and $x=0$, which cover all the situations. Numerical experiments show that the convergence of the proposed methods greatly improves when the frequency $\omega$ increases. Some ideas in this paper can also be extended to study oscillatory Hilbert transforms with Bessel oscillators. We give a brief description of this aspect in Section 4. We conclude this paper with some final remarks in Section 5 . 


\section{Asymptotic analysis of oscillatory Hilbert transforms}

\subsection{Large $\omega$ expansion with $x>0$}

We start with the derivation of asymptotic expansions of oscillatory Hilbert transforms (1.4) for large $\omega$ with $x>0$. An important ingredient in our analysis is the following lemma which allows us to reduce the Cauchy principal integrals (1.4) to ordinary integrals under certain restrictions on $f$.

Lemma 2.1. Let $f$ be a locally integrable function on $[0, \infty)$ and continuously differentiable over $(0, \infty)$. Suppose that $f$ has an analytic continuation in the first quadrant of the complex plane, except possibly a branch point at the origin, and there exist constants $M>0, \delta<1$ and $0 \leq d<\omega$ such that

$$
|f(z)| \leq M|z|^{\delta} e^{d \operatorname{Im}(z)},
$$

as $|z| \rightarrow \infty$ in the first quadrant. Then we have

$$
H^{+}\left(f(t) e^{i \omega t}\right)(x)=f_{0}^{\infty} e^{i \omega t} \frac{f(t)}{t-x} d t=i \pi e^{i \omega x} f(x)+\int_{0}^{\infty} e^{-\omega p} \frac{f(i p)}{p+i x} d p
$$

for each $x>0$, whenever the integral exists.

Proof. Let us consider a quarter of the disc centered at the origin with radius $R$, which lies in the first quadrant, and denote by $\Gamma_{R}$ its boundary, i.e.,

$$
\Gamma_{R}:=\{z|| z \mid=R, \operatorname{Re}(z)>0, \operatorname{Im}(z)>0\} .
$$

For each fixed $x>0$, we can find $R$ large enough such that a half disc $U_{x, \epsilon}^{+}:=\{z|| z-x \mid \leq$ $\epsilon, \operatorname{Im}(z)>0\}$ can be excluded from the quarter of the disc, where $\epsilon$ is a small positive number. The obtained domain is bounded by curves orientated in a counter-clockwise manner as illustrated in Figure 1 .

According to our assumptions on $f$, it is easily seen that the integrand $e^{i \omega t} \frac{f(t)}{t-x}$ of (1.4) is analytic in a quarter of the disc with a small indentation at $x$, as described above. We then obtain from Cauchy's theorem that

$$
\left(\int_{0}^{x-\epsilon}+\int_{\partial U_{x, \epsilon}^{+}}+\int_{x+\epsilon}^{R}+\int_{\Gamma_{R}}+\int_{i R}^{0}\right) e^{i \omega t} \frac{f(t)}{t-x} d t=0
$$

where $\partial U_{x, \epsilon}^{+}$stands for the boundary of $U_{x, \epsilon}^{+}$. This, together with (1.4), implies

$$
H^{+}\left(f(t) e^{i \omega t}\right)(x)=\lim _{\epsilon \rightarrow 0, R \rightarrow \infty}\left(-\int_{\Gamma_{R}}+\int_{0}^{i R}-\int_{\partial U_{x, \epsilon}^{+}}\right) e^{i \omega t} \frac{f(t)}{t-x} d t .
$$

We next evaluate the three integrals on the right hand side of (2.5).

A simple change of variable

$$
t=R e^{i \theta}, \quad 0 \leq \theta \leq \frac{\pi}{2},
$$




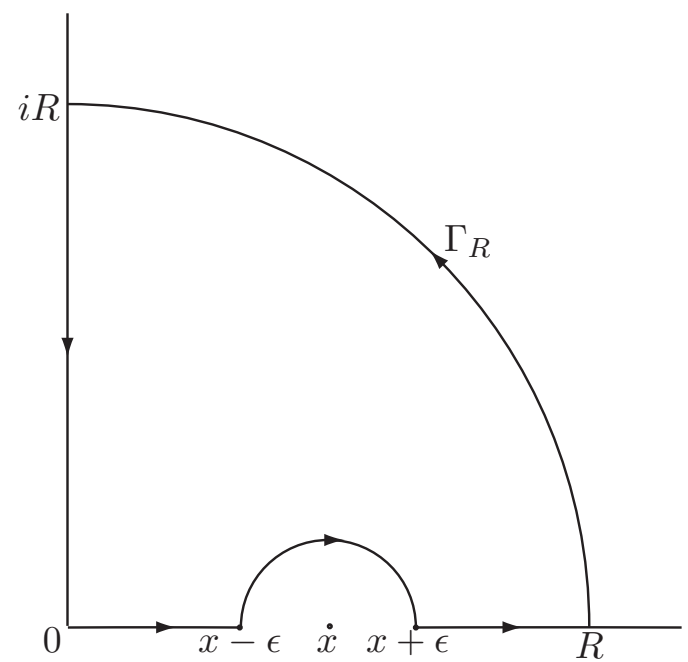

Figure 1: A quarter disc in the first quadrant with a small indentation at $x$.

yields

$$
\begin{aligned}
& \left|\int_{\Gamma_{R}} e^{i \omega t} \frac{f(t)}{t-x} d t\right|=\left|\int_{0}^{\frac{\pi}{2}} e^{i \omega R e^{i \theta}} \frac{f\left(R e^{i \theta}\right)}{R e^{i \theta}-x} R e^{i \theta} i d \theta\right| \\
& \leq R \int_{0}^{\frac{\pi}{2}} e^{-\omega R \sin \theta}\left|\frac{f\left(R e^{i \theta}\right)}{R e^{i \theta}-x}\right| d \theta \\
& \leq \frac{R}{R-x} \int_{0}^{\frac{\pi}{2}} e^{-\omega R \sin \theta}\left|f\left(R e^{i \theta}\right)\right| d \theta \\
& \leq \frac{M R^{1+\delta}}{R-x} \int_{0}^{\frac{\pi}{2}} e^{-(\omega-d) R \sin \theta} d \theta
\end{aligned}
$$

where in the last step we have made use of (2.1). Recall the well-known inequality $\sin \theta \geq \frac{2}{\pi} \theta$, if $0 \leq \theta \leq \frac{\pi}{2}$; cf. [1, p. 223]. For $R$ large enough, we obtain

$$
\begin{aligned}
\left|\int_{\Gamma_{R}} e^{i \omega t} \frac{f(t)}{t-x} d t\right| & \leq \frac{M R^{1+\delta}}{R-x} \int_{0}^{\frac{\pi}{2}} e^{-\frac{2}{\pi}(\omega-d) R \theta} d \theta, \\
& =\frac{\pi M R^{\delta}}{2(\omega-d)(R-x)}\left(1-e^{-(\omega-d) R}\right) \rightarrow 0, \quad \text { as } R \rightarrow \infty .
\end{aligned}
$$

It is also easily seen that

$$
\begin{aligned}
\lim _{R \rightarrow \infty} \int_{0}^{i R} e^{i \omega t} \frac{f(t)}{t-x} d t & =\lim _{R \rightarrow \infty} \int_{0}^{R} e^{-\omega p} \frac{f(i p)}{i p-x} i d p \\
& =\int_{0}^{\infty} e^{-\omega p} \frac{f(i p)}{p+i x} d p
\end{aligned}
$$


To evaluate the third integral over the contour $\partial U_{x, \epsilon}^{+}$, we appeal to [34, (2.7) and (2.9)], which gives

$$
\lim _{\epsilon \rightarrow 0} \int_{\partial U_{x, \epsilon}^{+}} e^{i \omega t} \frac{f(t)}{t-x} d t=-i \pi e^{i \omega x} f(x) .
$$

Finally, substituting (2.7)-(2.9) into (2.5), we obtain (2.2).

Now, we are ready to prove

Theorem 2.2. Let $f$ be a function as given in Lemma 2.1 and assume that $f$ takes an asymptotic expansion of the form

$$
f(t) \sim \sum_{j=0}^{\infty} a_{j} t^{j-\alpha}
$$

as $t \rightarrow 0^{+}$, where $0 \leq \alpha<1$. Then the one-sided oscillatory Hilbert transforms (1.4) can be expanded in the following fashion

$$
H^{+}\left(f(t) e^{i \omega t}\right)(x) \sim i \pi e^{i \omega x} f(x)-\sum_{\ell=0}^{\infty} \frac{\Gamma(\ell+1-\alpha)}{\omega^{\ell+1-\alpha}} e^{\frac{\pi}{2}(\ell+1-\alpha) i}\left(\sum_{j+k=\ell} \frac{a_{j}}{x^{k+1}}\right)
$$

as $\omega \rightarrow \infty$, for each $x>0$.

Proof. By (2.10), it follows that, for each fixed $x>0$,

$$
\begin{aligned}
\frac{f(i p)}{p+i x} & \sim \frac{1}{i x}\left(\sum_{j=0}^{\infty} a_{j}(i p)^{j-\alpha}\right)\left(\sum_{k=0}^{\infty} \frac{(i p)^{k}}{x^{k}}\right) \\
& =-i \sum_{\ell=0}^{\infty} i^{\ell-\alpha} p^{\ell-\alpha}\left(\sum_{j+k=\ell} \frac{a_{j}}{x^{k+1}}\right) \\
& =-\sum_{\ell=0}^{\infty} e^{\frac{\pi}{2}(\ell+1-\alpha) i} p^{\ell-\alpha}\left(\sum_{j+k=\ell} \frac{a_{j}}{x^{k+1}}\right)
\end{aligned}
$$

as $p \rightarrow 0^{+}$. In view of (2.2), an appeal to Watson's lemma (cf. [37, p. 20]) gives us,

$$
\begin{aligned}
H^{+}\left(f(t) e^{i \omega t}\right)(x) & =i \pi e^{i \omega x} f(x)+\int_{0}^{\infty} e^{-\omega p} \frac{f(i p)}{p+i x} d p \\
& \sim i \pi e^{i \omega x} f(x)-\sum_{\ell=0}^{\infty} e^{\frac{\pi}{2}(\ell+1-\alpha) i}\left(\sum_{j+k=\ell} \frac{a_{j}}{x^{k+1}}\right) \int_{0}^{\infty} p^{\ell-\alpha} e^{-\omega p} d p \\
& =i \pi e^{i \omega x} f(x)-\sum_{\ell=0}^{\infty} \frac{\Gamma(\ell+1-\alpha)}{\omega^{\ell+1-\alpha}} e^{\frac{\pi}{2}(\ell+1-\alpha) i}\left(\sum_{j+k=\ell} \frac{a_{j}}{x^{k+1}}\right),
\end{aligned}
$$

as $\omega \rightarrow \infty$, which is (2.11). 
Remark 2.3. It is worth noting that the expansion (2.11) is only uniformly valid for $x$ bounded away from 0 . To clarify the behavior when $0<x \ll 1$, we make the following decomposition:

$$
\begin{aligned}
f_{0}^{\infty} \frac{f(t)}{t-x} e^{i \omega t} d t & =f_{0}^{\infty} \frac{f_{\alpha}(t)}{t^{\alpha}(t-x)} e^{i \omega t} d t \\
& =\int_{0}^{\infty} \frac{f_{\alpha}(t)-f_{\alpha}(x)}{t^{\alpha}(t-x)} e^{i \omega t} d t+f_{\alpha}(x) f_{0}^{\infty} \frac{e^{i \omega t}}{t^{\alpha}(t-x)} d t
\end{aligned}
$$

with $f_{\alpha}(t)=t^{\alpha} f(t)$. For the first integral on the right hand side of (2.13), in view of the expansion

$$
\frac{f_{\alpha}(t)-f_{\alpha}(x)}{t-x} \sim\left(\sum_{j=1}^{\infty} a_{j} x^{j-1}\right)+\left(\sum_{j=2}^{\infty} a_{j} x^{j-1}\right) t+\mathcal{O}\left(t^{2}\right)
$$

as $t \rightarrow 0^{+}$, then by [35, Thm. 1, p. 199] we find that

$$
\int_{0}^{\infty} \frac{f_{\alpha}(t)-f_{\alpha}(x)}{t^{\alpha}(t-x)} e^{i \omega t} d t \sim\left(\sum_{j=1}^{\infty} a_{j} x^{j-1}\right) \frac{\Gamma(1-\alpha) e^{\frac{\pi}{2}(1-\alpha) i}}{\omega^{1-\alpha}}+\mathcal{O}\left(\frac{1}{\omega^{2-\alpha}}\right), \quad \omega \rightarrow \infty
$$

where the sum on the right side converges when $0<x \ll 1$. For the second one, we obtain from [35] and (3.6) below that

$$
f_{0}^{\infty} \frac{e^{i \omega t}}{t^{\alpha}(t-x)} d t= \begin{cases}\frac{e^{i \omega x}}{x^{\alpha}}\left[e^{-i \alpha \pi} \Gamma(1-\alpha) \Gamma(\alpha, i \omega x)+i \pi\right], & \text { if } 0<\alpha<1 \\ e^{i \omega x}[\operatorname{Ei}(1, i \omega x)+i \pi], & \text { if } \alpha=0 .\end{cases}
$$

Combining these results, we can see clearly that the asymptotic behaviour of the oscillatory Hilbert transforms (1.4) depends strongly on the behaviour of the product of $\omega$ and $x$. For example, the asymptotic behaviour of (1.4) as $x \rightarrow 0^{+}, \omega \rightarrow \infty$ and $\omega x \rightarrow 0^{+}$can be derived by taking into account the asymptotic expansions of $\operatorname{Ei}(1, i \omega x)$ and $\Gamma(\alpha, i \omega x)$ respectively. We omit the details here and only give a leading term

$$
f_{0}^{\infty} \frac{f(t)}{t-x} e^{i \omega t} d t \sim \begin{cases}\frac{a_{0}}{x^{\alpha}}\left[\frac{\pi}{\sin (\alpha \pi)} e^{-i \alpha \pi}+i \pi\right]+\mathcal{O}\left(\omega^{\alpha}\right), & \text { if } 0<\alpha<1 \\ -a_{0} \log (\omega x)+\mathcal{O}(1), & \text { if } \alpha=0\end{cases}
$$

Remark 2.4. In Theorem 2.2, we require that $f$ has an analytic continuation in the first quadrant and satisfies the growth condition (2.1). These conditions can be further relaxed to allow $f$ has a simple pole in the first quadrant or $f$ only has an analytic continuation around the real axis and satisfies (2.1). In both cases, the only contribution will be a exponentially small term in $\omega$, thus, the expansion (2.11) still holds. 


\subsection{Large $\omega$ expansion with $x=0$ : asymptotics of Hadamard finite-part integrals}

When $x=0$, the integrand of the one-sided oscillatory Hilbert transforms (1.4) has a singularity at the origin. If the integral is divergent, the transform should be understood as a finite-part integral in the Hadamard sense. Note that the definition of Hadamard finite-part integral does not change the value of a convergent integral. It is the aim of this section to find the asymptotics of (1.4) with $x=0$.

Assume that $f$ still admits an asymptotic expansion near the origin as given in (2.10), we then formally have

$$
\int_{0}^{\infty} e^{i \omega t} \frac{f(t)}{t} d t=a_{0} \int_{0}^{\infty} \frac{e^{i \omega t}}{t^{\alpha+1}} d t+\int_{0}^{\infty} e^{i \omega t} \frac{f(t)-a_{0} t^{-\alpha}}{t} d t
$$

There are two integrals on the right hand side of (2.16). The first one is divergent and should be interpreted as a Hadamard finite-part integral over the positive real axis. The integrand of the second one has an integrable singularity at the origin. Hence, it is well defined.

To this end, one needs to extend standard Hadamard finite-part integrals for the finite interval (cf. [19, Sec. 1.4]) to semifinite integrals. Here, we adapt the definition from [25]:

Definition 2.5. Let $g(x)$ be of class $C^{m+1}[0, \infty)$ and such that

$$
\left|\int_{0}^{\infty} g^{(k)}(t) t^{p-1} d t\right|<\infty, \quad k=0, \ldots, m+1
$$

for all $p \geq 1$. Then for any $\eta \geq 1$, a finite-part integral of order $\delta$ for the positive real axis is defined as

$$
f_{0}^{\infty} \frac{g(t)}{t^{\eta}} d t:=f_{0}^{b} \frac{g(t)}{t^{\eta}} d t+\int_{b}^{\infty} \frac{g(t)}{t^{\eta}} d t
$$

where $f$ stands for a Hadamard finite-part integral and $b$ is an arbitrary positive constant.

In [25], a function $g$ satisfying (2.17) is called allowable. The Definition 2.5 defines a Hadamard finite-part integral for the infinite interval from a finite-part integral on the finite interval, and it is independent of the choice of $b$.

With Definition 2.5. we observe that

Lemma 2.6.

$$
f_{0}^{\infty} \frac{e^{i \omega t}}{t^{\alpha+1}} d t= \begin{cases}\frac{e^{\frac{\pi}{2}(2-\alpha) i} \omega^{\alpha}}{\alpha} \Gamma(1-\alpha), & \text { if } 0<\alpha<1, \\ -\gamma-\log \omega+i \frac{\pi}{2}, & \text { if } \alpha=0\end{cases}
$$

where $\gamma$ is the Euler constant. 
Proof. From (2.18), it is readily seen that

$$
f_{0}^{\infty} \frac{e^{i \omega t}}{t^{\alpha+1}} d t:=f_{0}^{b} \frac{e^{i \omega t}}{t^{\alpha+1}} d t+\int_{b}^{\infty} \frac{e^{i \omega t}}{t^{\alpha+1}} d t
$$

where $b$ is an arbitrary positive constant. If $0<\alpha<1$, an appeal to integration by parts gives us

$$
\begin{aligned}
\int_{\varepsilon}^{b} \frac{e^{i \omega t}}{t^{\alpha+1}} d t & =\frac{e^{i \omega \varepsilon}}{\alpha \varepsilon^{\alpha}}-\frac{e^{i \omega b}}{\alpha b^{\alpha}}+\frac{i \omega}{\alpha} \int_{\varepsilon}^{b} t^{-\alpha} e^{i \omega t} d t \\
& =\frac{e^{i \omega \varepsilon}}{\alpha \varepsilon^{\alpha}}-\frac{e^{i \omega b}}{\alpha b^{\alpha}}+\frac{i \omega}{\alpha}\left(\frac{\Gamma(1-\alpha)}{(-i \omega)^{1-\alpha}}-b^{1-\alpha} \operatorname{Ei}(\alpha,-i \omega b)-\int_{0}^{\varepsilon} t^{-\alpha} e^{i \omega t} d t\right)
\end{aligned}
$$

and

$$
\int_{b}^{\infty} \frac{e^{i \omega t}}{t^{\alpha+1}} d t=\frac{e^{i \omega b}}{\alpha b^{\alpha}}+\frac{i \omega}{\alpha} \int_{b}^{\infty} t^{-\alpha} e^{i \omega t} d t=\frac{e^{i \omega b}}{\alpha b^{\alpha}}+\frac{i \omega}{\alpha} b^{1-\alpha} \operatorname{Ei}(\alpha,-i \omega b),
$$

where $\varepsilon$ is a small positive number and

$$
\operatorname{Ei}(\rho, z):=\int_{1}^{\infty} t^{-\rho} e^{-z t} d t, \quad \rho>0, \quad \operatorname{Re}(z) \geq 0
$$

is the exponential integral. In (2.21), by neglecting the divergent term $\frac{e^{i \omega \varepsilon}}{\alpha \varepsilon^{\alpha}}$ and noting that the last integral vanishes as $\varepsilon \rightarrow 0^{+}$, we obtain

$$
f_{0}^{b} \frac{e^{i \omega t}}{t^{\alpha+1}} d t:=-\frac{e^{i \omega b}}{\alpha b^{\alpha}}+\frac{i \omega}{\alpha}\left(\frac{\Gamma(1-\alpha)}{(-i \omega)^{1-\alpha}}-b^{1-\alpha} \operatorname{Ei}(\alpha,-i \omega b)\right) .
$$

Combining (2.20), (2.24) and (2.22), we get the desired result.

Similarly, if $\alpha=0$, we note that

$$
\left(\int_{\varepsilon}^{b}+\int_{b}^{\infty}\right) \frac{e^{i \omega t}}{t} d t=\operatorname{Ei}(1,-i \omega \varepsilon)=-\gamma-\log \omega+i \frac{\pi}{2}-\log \varepsilon+\mathcal{O}(\varepsilon)
$$

as $\varepsilon \rightarrow 0$, where $\gamma$ is the Euler constant. Hence,

$$
f_{0}^{\infty} \frac{e^{i \omega t}}{t} d t:=-\gamma-\log \omega+i \frac{\pi}{2}
$$

as shown in (2.19).

A combination of Lemma 2.6 and (2.16) gives us

$$
f_{0}^{\infty} e^{i \omega t} \frac{f(t)}{t} d t= \begin{cases}\frac{e^{\frac{\pi}{2}(2-\alpha) i} \omega^{\alpha}}{\alpha} \Gamma(1-\alpha) a_{0}+\int_{0}^{\infty} e^{i \omega t} \frac{f(t)-a_{0} t^{-\alpha}}{t} d t, & \text { if } 0<\alpha<1 \\ \left(-\gamma-\log \omega+i \frac{\pi}{2}\right) f(0)+\int_{0}^{\infty} e^{i \omega t} \frac{f(t)-f(0)}{t} d t, & \text { if } \alpha=0 .\end{cases}
$$


Here we have made use of the fact that $a_{0}=f(0)$ if $\alpha=0$ in (2.10). To derive asymptotic expansions of Hadamard finite part integrals (2.27), it is then sufficient to find asymptotics of Fourier-type integrals $\int_{0}^{\infty} \tilde{f}(t) e^{i \omega t} d t$, where

$$
\tilde{f}(t):=\frac{f(t)-a_{0} t^{-\alpha}}{t}, \quad 0 \leq \alpha<1 .
$$

Our result is stated below:

Theorem 2.7. Let $f$ be a locally integrable function on $[0, \infty)$ and $m$ times continuously differentiable over $(0, \infty), m$ being a positive integer. Suppose that $f$ has the asymptotic expansion (2.10), and this expansion can be differentiated $m$ times. Moreover, each of the integrals

$$
\int_{1}^{\infty} \tilde{f}^{(s)}(t) e^{i \omega t} d t, \quad s=0,1, \cdots, m
$$

converges uniformly for $\omega$ sufficiently large, where $\tilde{f}$ is given in (2.28). Then, as $\omega \rightarrow \infty$, we have

$f_{0}^{\infty} e^{i \omega t} \frac{f(t)}{t} d t= \begin{cases}\frac{e^{\frac{\pi}{2}(2-\alpha) i} \omega^{\alpha}}{\alpha} \Gamma(1-\alpha) a_{0}+\sum_{\ell=1}^{m} a_{\ell} e^{\frac{\pi}{2}(\ell-\alpha) i} \frac{\Gamma(\ell-\alpha)}{\omega^{\ell-\alpha}}+o\left(\omega^{-m}\right), & \text { if } 0<\alpha<1, \\ \left(i \frac{\pi}{2}-\gamma-\log \omega\right) f(0)+\sum_{\ell=1}^{m} a_{\ell} e^{\frac{\pi \ell}{2}} \frac{(\ell-1) !}{\omega^{\ell}}+o\left(\omega^{-m}\right), & \text { if } \alpha=0 .\end{cases}$

Proof. From (2.28) and (2.10), it is readily seen that

$$
\tilde{f}(t)=\frac{f(t)-a_{0} t^{-\alpha}}{t} \sim \sum_{j=0}^{\infty} a_{j+1} t^{j-\alpha}
$$

as $t \rightarrow 0^{+}$. This, together with [35, Thm. 1, p. 199], leads us to the following asymptotics of Fourier integrals:

$$
\int_{0}^{\infty} \tilde{f}(t) e^{i \omega t}=\sum_{\ell=1}^{m-1} a_{\ell} e^{\frac{\pi}{2}(\ell-\alpha) i} \frac{\Gamma(\ell-\alpha)}{\omega^{\ell-\alpha}}+o\left(\omega^{-m}\right) .
$$

The asymptotic expansions (2.30) of Hadamard finite-part integrals then follows from a combination of (2.27) and (2.32).

Remark 2.8. We do not require that $f$ has an analytic continuation to the first quadrant in Theorem 2.7. However, such requirement is essential in the proof of Theorem 2.2.

\subsection{Some examples}

We conclude this section with applications of Theorems 2.2 and 2.7 to some concrete examples. 
Example 2.9. Consider the integral

$$
f_{0}^{\infty} e^{i \omega t} \frac{e^{-c t}}{t-x} d t, \quad c \geq 0 .
$$

This is the one-sided oscillatory Hilbert transform of $f(t)=e^{-c t}$. Clearly, $f$ is an entire function and satisfies all the assumptions of our theorems. Since

$$
f(t) \sim \sum_{j=0}^{\infty} \frac{(-c)^{j}}{j !} t^{j}
$$

as $t \rightarrow 0^{+}$, one has

$$
\alpha=0, \quad a_{j}=\frac{(-c)^{j}}{j !}, \quad j=0,1,2, \cdots,
$$

in the notation of (2.10). Hence, from (2.11) and (2.30), it is easily seen that

$$
f_{0}^{\infty} e^{i \omega t} \frac{e^{-c t}}{t-x} d t \sim i \pi e^{i \omega x} e^{-c x}-\sum_{\ell=0}^{\infty} \frac{\Gamma(\ell+1)}{\omega^{\ell+1}} e^{\frac{\pi}{2}(\ell+1) i}\left(\sum_{j+k=\ell} \frac{(-c)^{j}}{j ! x^{k+1}}\right), \quad x>0,
$$

and

$$
f_{0}^{\infty} e^{i \omega t} \frac{e^{-c t}}{t} d t \sim i \frac{\pi}{2}-\gamma-\log \omega+\sum_{\ell=1}^{\infty} \frac{(-c)^{\ell}}{\ell} e^{i \frac{\pi}{2} \ell} \frac{1}{\omega^{\ell}},
$$

as $\omega \rightarrow \infty$. In particular, if $c=0$, i.e., $f \equiv 1$, the above two expansions can be simplified, and we have

$$
f_{0}^{\infty} \frac{e^{i \omega t}}{t-x} d t \sim i \pi e^{i \omega x}-\sum_{\ell=0}^{\infty} \frac{\Gamma(\ell+1)}{(\omega x)^{\ell+1}} e^{\frac{\pi}{2}(\ell+1) i}, \quad x>0
$$

and

$$
f_{0}^{\infty} \frac{e^{i \omega t}}{t} d t=i \frac{\pi}{2}-\gamma-\log \omega
$$

It is worthwhile to point out that the asymptotic expansion (2.38) is the same as the large $x$ expansion of $f_{0}^{\infty} \frac{e^{i \omega t}}{t-x} d t$ with $\omega>0$; see [32, Lem. 1].

Example 2.10. As the second example, we consider

$$
f_{0}^{\infty} e^{i \omega t} \frac{\sqrt{t}}{(1+t)(t-x)} d t
$$

Thus, $f(t)=\sqrt{t} /(1+t)$, which can be analytically extended to the complex plane with a pole at -1 and a branch cut along the negative axis. As $t \rightarrow 0^{+}$, we have

$$
f(t) \sim \sqrt{t} \sum_{j=0}^{\infty}(-t)^{j}=\sum_{j=1}^{\infty}(-1)^{j+1} t^{j-\frac{1}{2}} .
$$


Hence, in the notation of (2.10) $, \alpha=1 / 2, a_{0}=0, a_{j}=(-1)^{j+1}, j=1,2, \cdots$. An appeal to (2.11) and (2.30) then gives

$$
f_{0}^{\infty} e^{i \omega t} \frac{\sqrt{t}}{(1+t)(t-x)} d t \sim i \pi e^{i \omega x} \frac{\sqrt{x}}{1+x}-\sum_{\ell=1}^{\infty} \frac{\Gamma\left(\ell+\frac{1}{2}\right)}{\omega^{\ell+\frac{1}{2}}} e^{\frac{\pi}{2}\left(\ell+\frac{1}{2}\right) i}\left(\sum_{j=1}^{\ell} \frac{(-1)^{j+\ell}}{x^{j}}\right)
$$

for $x>0$, and

$$
f_{0}^{\infty} e^{i \omega t} \frac{\sqrt{t}}{t(1+t)} d t \sim \sum_{\ell=1}^{\infty}(-1)^{\ell+1} e^{\frac{\pi}{2}\left(\ell-\frac{1}{2}\right) i} \frac{\Gamma\left(\ell-\frac{1}{2}\right)}{\omega^{\ell-\frac{1}{2}}}
$$

\section{Computation of oscillatory Hilbert transforms}

Although the asymptotic expansions derived in the previous section provide essential insights into the behaviour of the oscillatory Hilbert transforms for large $\omega$, they are not suitable for computational purpose, since asymptotic expansions are typically divergent, one can not simply keep on adding terms of the expansion in order to improve the accuracy of the approximation. In this section we shall focus on fast numerical computation of oscillatory Hilbert transforms (1.4). According to the position of $x$, we classify our discussion into three regimes, namely, $x=\mathcal{O}(1)$ or $x \gg 1,0<x \ll 1$ and $x=0$. Since these regimes exhibit different asymptotic behaviour, they also require different numerical methods.

\subsection{The regime $x=\mathcal{O}(1)$ or $x \gg 1$}

If $x$ is not so close to the origin, say, $x=\mathcal{O}(1)$ or $x \gg 1$, the one-sided oscillatory Hilbert transform (1.4) can be approximated efficiently using the generalized Gauss-Laguerre quadrature rule. To see this, we observe from (2.2) that

$$
\begin{aligned}
H^{+}\left(f(t) e^{i \omega t}\right)(x) & =i \pi f(x) e^{i \omega x}+\frac{1}{\omega} \int_{0}^{\infty} e^{-q} \frac{f\left(\frac{i q}{\omega}\right)}{\frac{q}{\omega}+i x} d q \\
& =i \pi f(x) e^{i \omega x}+\frac{\exp \left(-\frac{\alpha}{2} \pi i\right)}{\omega^{1-\alpha}} \int_{0}^{\infty} q^{-\alpha} e^{-q} \frac{f_{\alpha}\left(\frac{i q}{\omega}\right)}{\frac{q}{\omega}+i x} d q,
\end{aligned}
$$

with $f_{\alpha}(t)=t^{\alpha} f(t)$ and where $\alpha$ is defined as in (2.10). We have made use of the change of variable $q=\omega p$ in the first equality. On account of (2.10), it is easily seen that $f_{\alpha}\left(\frac{i q}{\omega}\right) /\left(\frac{q}{\omega}+i x\right)$ behaves like a polynomial near the origin, if $x=\mathcal{O}(1)$ or $x \gg 1$. This is exactly the situation that can be handled by the generalized Gauss-Laguerre quadrature rule. Let $\left\{t_{j}, w_{j}\right\}_{j=1}^{n}$ be the nodes and weights of the generalized GaussLaguerre quadrature rule with respect to the weight $t^{-\alpha} e^{-t}$, with $0 \leq \alpha<1$. Then the one-sided oscillatory Hilbert transform $H^{+}\left(f(t) e^{i \omega t}\right)(x)$ is approximated by

$$
Q_{n}(f, \omega, x)=i \pi f(x) e^{i \omega x}+\frac{\exp \left(-\frac{\alpha}{2} \pi i\right)}{\omega^{1-\alpha}} \sum_{k=1}^{n} w_{k} \frac{f_{\alpha}\left(\frac{i t_{k}}{\omega}\right)}{\frac{t_{k}}{\omega}+i x} .
$$


Applying the error expression of the $n$-point generalized Gauss-Laguerre quadrature rule [10. p. 223], we can estimate, for each fixed $x$, the quadrature error as follows:

$$
\begin{aligned}
H^{+}\left(f(t) e^{i \omega t}\right)(x)-Q_{n}(f, \omega, x) & =\frac{\exp \left(-\frac{\alpha}{2} \pi i\right)}{\omega^{1-\alpha}}\left(\int_{0}^{\infty} q^{-\alpha} e^{-q} \frac{f_{\alpha}\left(i \frac{q}{\omega}\right)}{\frac{q}{\omega}+i x} d q-\sum_{k=1}^{n} w_{k} \frac{f_{\alpha}\left(\frac{i t_{k}}{\omega}\right)}{\frac{t_{k}}{\omega}+i x}\right) \\
& =\left.\frac{\exp \left(-\frac{\alpha}{2} \pi i\right)}{\omega^{1-\alpha}} \frac{n ! \Gamma(n-\alpha+1)}{(2 n) !}\left(\frac{f_{\alpha}\left(i \frac{q}{\omega}\right)}{\frac{q}{\omega}+i x}\right)^{(2 n)}\right|_{q=\xi} \\
& =\mathcal{O}\left(\omega^{-2 n-1+\alpha}\right)
\end{aligned}
$$

for some constant $\xi \in \mathbb{C}$ as $\omega \rightarrow \infty$. Note that one gains the factor $\omega^{-2 n}$ from taking the $(2 n)$-th order derivative of a function of $q$ that depends only on $q / \omega$. We observe that the accuracy of the quadrature rule (3.2) rapidly improves as $\omega$ grows. This result may come as a surprise here, since we started out with a highly oscillatory integral that typically requires a growing number of quadrature points to evaluate as the frequency increases. Yet, we like to point out that this result is entirely parallel to the case of finite Fourier integrals of the form (1.1), where an application of Gauss-Laguerre yields similar behaviour for large $\omega$. In what follows, we give several examples to illustrate the convergence of our quadrature rule $Q_{n}(f, \omega, x)$. Throughout this paper, all the computations have been performed using Maple 14 with 32-digit arithmetif*.

Example 3.1. Let us consider (2.33) with $c=1$, that is, $f(t)=e^{-t}$, and one has $\alpha=0$ in (3.2). The exact solution of (2.33) can be obtained by using the definition of the Cauchy principal value integral and taking the series expansion of the exponential function. The result is

$$
\begin{aligned}
& f_{0}^{\infty} e^{i \omega t} \frac{e^{-c t}}{t-x} d t \\
& =e^{(-c+i \omega) x}\left[\operatorname{Ei}(1,(c-i \omega) x)-2 \sum_{\ell=0}^{\infty} \frac{c^{2 \ell+1}}{(2 \ell+1) !}\left(\sum_{k=0}^{2 \ell} k !\left(\begin{array}{c}
2 \ell \\
k
\end{array}\right) \frac{x^{2 \ell-k}}{\omega^{k+1}} \sin \left(\omega x+\frac{k \pi}{2}\right)\right)\right. \\
& \left.\quad+i\left\{2 \operatorname{Si}(\omega x)-2 \sum_{\ell=0}^{\infty} \frac{c^{2 \ell+2}}{(2 \ell+2) !}\left(\sum_{k=0}^{2 \ell+1} k !\left(\begin{array}{c}
2 \ell+1 \\
k
\end{array}\right) \frac{x^{2 \ell+1-k}}{\omega^{k+1}} \cos \left(\omega x+\frac{k \pi}{2}\right)\right)\right\}\right]
\end{aligned}
$$

where

$$
\operatorname{Si}(x)=\int_{0}^{x} \frac{\sin t}{t} d t, \quad x \geq 0,
$$

is the sine integral; cf. [2]. In the case of $c=0$, one can check that

$$
f_{0}^{\infty} \frac{e^{i \omega t}}{t-x} d t=e^{i \omega x}(\operatorname{Ei}(1,-i \omega x)+i 2 \operatorname{Si}(\omega x))=e^{i \omega x}(i \pi+\operatorname{Ei}(1, i \omega x)) .
$$

We compute the error for $x=1$ and $x=5$ with different frequency $\omega$ and $n$ ranging from 2 to 10. The results are illustrated in Figure 2 .

\footnotetext{
${ }^{*}$ The use of increased precision is just to show the convergence rates of our methods.
} 

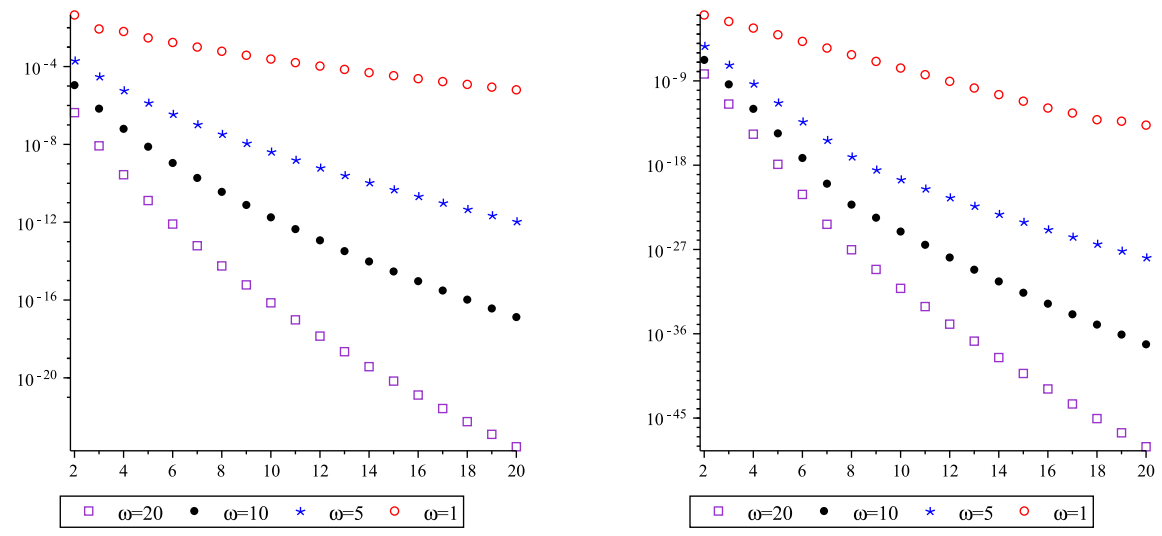

Figure 2: The error of the quadrature $Q_{n}(f, \omega, x)$ for $x=1$ (left) and $x=5$ (right) with $f(t)=e^{-t}$ and $n$ ranging from 2 to 10 .

Example 3.2. We next consider the function $f(t)=\frac{\cos t}{\sqrt[3]{t}}$, which grows exponentially in the complex plane. We can check that it satisfies (2.1) with $d=1$, and $\alpha=\frac{1}{3}$ in (3.2). The error is illustrated in Figure 3 .
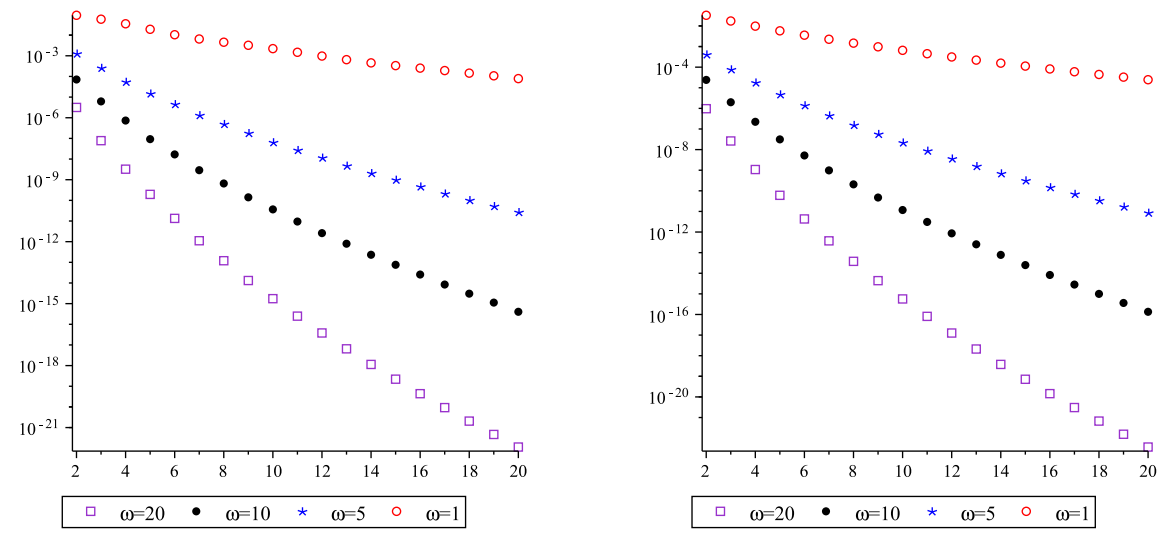

Figure 3: The error of the quadrature $Q_{n}(f, \omega, x)$ for $x=1$ (left) and $x=5$ (right) with $f(t)=\frac{\cos t}{\sqrt[3]{t}}$ and $n$ ranging from 2 to 10 .

Example 3.3. We return to Example 2.10, i.e., $f(t)=\sqrt{t} /(1+t)$, which corresponds to $\alpha=1 / 2$. Numerical results are displayed in Figure 4

All these examples show that the accuracy of quadrature rule (3.2) improves rapidly as $n$ increases. Meanwhile, the convergence is faster for larger $\omega$. 

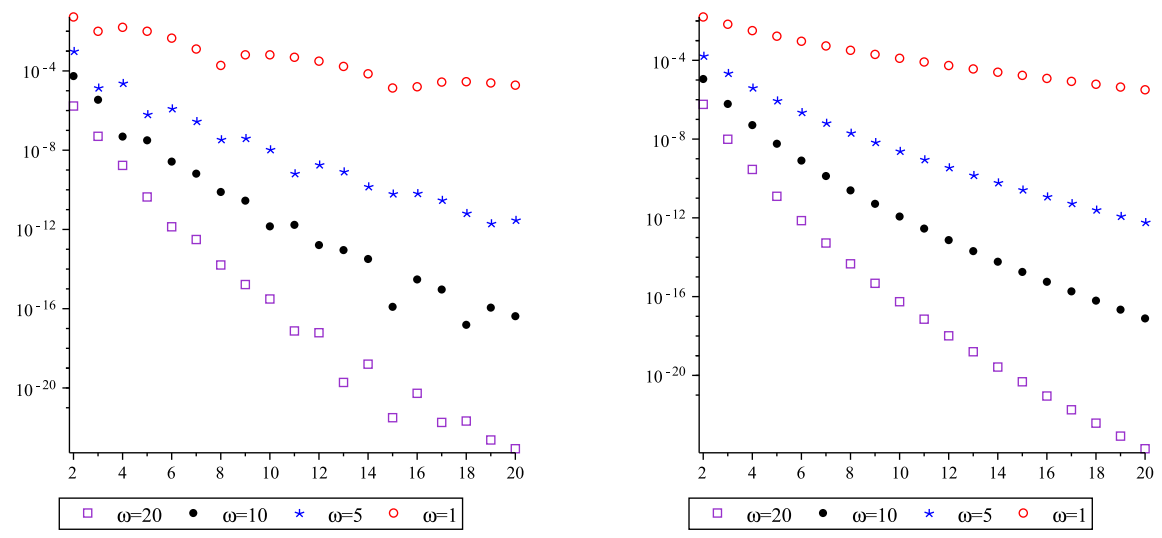

Figure 4: The error of the quadrature $Q_{n}(f, \omega, x)$ for $x=1$ (left) and $x=5$ (right) with $f(t)=\frac{\sqrt{t}}{1+t}$ and $n$ ranging from 2 to 10 .

\subsection{The regime $0<x \ll 1$}

When $x$ is close the origin, i.e., $0<x \ll 1$, the accuracy of the generalized GaussLaguerre rule deteriorates since the integrand on the right hand side of (3.1) is nearly singular. To this end, we make the following decomposition of oscillatory Hilbert transforms:

$$
H^{+}\left(f(t) e^{i \omega t}\right)(x)=\int_{0}^{a} \frac{f(t)}{t-x} e^{i \omega t} d t+\int_{a}^{\infty} \frac{f(t)}{t-x} e^{i \omega t} d t,
$$

where $a$ is a positive number larger than $x$. Let the two integrals on the right hand side of (3.7) be denoted by $I_{1}(x)$ and $I_{2}(x)$, respectively. Note that the integral $I_{2}(x)$ is no longer singular. A fast computation of oscillatory Hilbert transforms in this regime is then reduced to the numerical study of $I_{1}(x)$ and $I_{2}(x)$, which will be discussed in the next two sections.

\subsubsection{Computation of $I_{1}(x)$}

The integral $I_{1}(x)$ is a finite oscillatory Hilbert transform. By scaling the interval of integration to $(-1,1)$, we have

$$
\begin{aligned}
I_{1}(x) & =f_{0}^{a} \frac{f(t)}{t-x} e^{i \omega t} d t \\
& =e^{i \tilde{\omega}} f_{-1}^{1} \frac{f\left(\frac{a}{2}(y+1)\right)}{y-\tau} e^{i \tilde{\omega} y} d y \\
& =\left(\frac{a}{2}\right)^{-\alpha} e^{i \tilde{\omega}} f_{-1}^{1} \frac{(y+1)^{-\alpha} h(y)}{y-\tau} e^{i \tilde{\omega} y} d y,
\end{aligned}
$$

where $\tilde{\omega}=\frac{\omega a}{2}, \tau=\frac{2 x}{a}-1 \in(-1,1)$, and

$$
h(y)=f_{\alpha}\left(\frac{a}{2}(y+1)\right)=\left(\frac{a}{2}(y+1)\right)^{\alpha} f\left(\frac{a}{2}(y+1)\right) .
$$


The computation of (3.8) with $\alpha=0$ has been discussed in [33. Let $p_{N}(y)$ be the Lagrange polynomial which interpolates $h(y)$ at the Clenshaw-Curtis points $y_{j}=$ $\cos \left(\frac{j \pi}{N}\right), j=0, \ldots, N$. Then one has (see [6])

$$
p_{N}(y)=\sum_{k=0}^{N}{ }^{\prime \prime} a_{k}^{N} T_{k}(y)
$$

with

$$
a_{k}^{N}=\frac{2}{N} \sum_{j=0}^{N} " h\left(y_{j}\right) T_{j}\left(y_{k}\right), \quad k=0, \ldots, N,
$$

where the double prime denotes a sum whose first and last terms are halved and $T_{j}(y)$ is the Chebyshev polynomial of the first kind of degree $j$. The coefficients $a_{k}^{N}$ can be computed efficiently by FFT [11. Replacing $h(y)$ in (3.8) by $p_{N}(y)$, we have

$$
\begin{aligned}
f_{-1}^{1} \frac{f(y)}{y-\tau} e^{i \tilde{\omega} y} d y & \simeq f_{-1}^{1} \frac{p_{N}(y)}{y-\tau} e^{i \tilde{\omega} y} d y \\
& =f_{-1}^{1} \frac{p_{N}(y)-p_{N}(\tau)}{y-\tau} e^{i \tilde{\omega} y} d y+p_{N}(\tau) f_{-1}^{1} \frac{e^{i \tilde{\omega} y}}{y-\tau} d y \\
& =2 \sum_{k=0}^{N}{ }^{\prime \prime} a_{k}^{N}\left[\sum_{n=0}^{k-1} T_{n}(\tau) M_{k-1-n}\right]+p_{N}(\tau) f_{-1}^{1} \frac{e^{i \tilde{\omega} y}}{y-\tau} d y
\end{aligned}
$$

where

$$
M_{n}=\int_{-1}^{1} U_{n}(y) e^{i \tilde{\omega} y} d y, \quad n \geq 0,
$$

and where $U_{n}(y)$ is the Chebyshev polynomial of the second kind of degree $n$. The last integral in (3.12) can be computed in closed form and the $M_{n}$ can be computed by using a three-term recurrence relation. The advantage of this method is that it converges uniformly with respect to $\tau \in(-1,1)$ as $N \rightarrow \infty$, provided $h(y)$ is analytic in a small neighborhood containing $[-1,1]$. However, this method costs $\mathcal{O}\left(N^{2}\right)$ operations which make it inefficient for large $N$.

In the following we shall present a more efficient implementation, which costs only $\mathcal{O}\left(N \log _{2} N\right)$ operations, to compute the finite oscillatory Hilbert transform (3.8). The key observation is that we can write $\left(p_{N}(y)-p_{N}(\tau)\right) /(y-\tau)$ in terms of $T_{k}(y)$ and this process can be performed in only $\mathcal{O}(N)$ operations.

We approximate $I_{1}(x)$ by

$$
\begin{aligned}
& Q_{N}^{(1)}(f, \tilde{\omega}, x)=\left(\frac{a}{2}\right)^{-\alpha} e^{i \tilde{\omega}}\left(f_{-1}^{1}(y+1)^{-\alpha} \frac{p_{N}(y)}{y-\tau} e^{i \tilde{\omega} y} d y\right) \\
&=\left(\frac{a}{2}\right)^{-\alpha} e^{i \tilde{\omega}}\left(\int_{-1}^{1}(y+1)^{-\alpha} \frac{p_{N}(y)-p_{N}(\tau)}{y-\tau} e^{i \tilde{\omega} y} d y\right. \\
&\left.\quad+p_{N}(\tau) f_{-1}^{1}(y+1)^{-\alpha} \frac{e^{i \tilde{\omega} y}}{y-\tau} d y\right) .
\end{aligned}
$$


We next expand $\left(p_{N}(y)-p_{N}(\tau)\right) /(y-\tau)$ in terms of $T_{k}(y)$ and obtain

$$
\frac{p_{N}(y)-p_{N}(\tau)}{y-\tau}=\sum_{k=0}^{N-1}{ }^{\prime} b_{k}^{N} T_{k}(y)
$$

where the prime denotes the summation whose first term is halved. The coefficients $b_{k}^{N}$ satisfy a three-term recurrence relation

$$
b_{k-1}^{N}=2 a_{k}^{N}+2 \tau b_{k}^{N}-b_{k+1}^{N}, \quad k=N-1, \ldots, 1,
$$

and the first two initial values are given by $b_{N}^{N}=0$ and $b_{N-1}^{N}=a_{N}^{N}$; see [13]. Inserting (3.14) into (3.13) gives us

$$
Q_{N}^{(1)}(f, \tilde{\omega}, x)=\left(\frac{a}{2}\right)^{-\alpha} e^{i \tilde{\omega}}\left(\sum_{k=0}^{N-1}{ }^{\prime} b_{k}^{N} Z_{k}^{(\alpha)}+p_{N}(\tau) f_{-1}^{1}(y+1)^{-\alpha} \frac{e^{i \tilde{\omega} y}}{y-\tau} d y\right),
$$

where

$$
Z_{k}^{(\alpha)}:=Z_{k}(\alpha, \tilde{\omega})=\int_{-1}^{1}(y+1)^{-\alpha} T_{k}(y) e^{i \tilde{\omega} y} d y, \quad k \geq 0
$$

Now, we only need to compute the moments $Z_{k}^{(\alpha)}$ and the integral on right hand side of (3.16) efficiently. It turns out that $Z_{k}^{(\alpha)}$ satisfy the following four-term recurrence relation (see [31]):

$$
\begin{aligned}
& i \tilde{\omega}(n-1) Z_{n+1}^{(\alpha)}+[2(n-\alpha+1)(n-1)+i \tilde{\omega}(n-2)] Z_{n}^{(\alpha)} \\
& \quad+[2 n(n+\alpha-2)-i \tilde{\omega}(n+1)] Z_{n-1}^{(\alpha)}-i \tilde{\omega} n Z_{n-2}^{(\alpha)}=-2^{2-\alpha} e^{i \tilde{\omega}}
\end{aligned}
$$

with the first three initial values given by

$$
\begin{aligned}
& Z_{0}^{(\alpha)}=\frac{e^{-i \tilde{\omega}}}{\tilde{\omega}^{1-\alpha}} e^{\frac{1}{2} \pi i(1-\alpha)} \gamma(1-\alpha,-2 i \tilde{\omega}), \\
& Z_{1}^{(\alpha)}=\frac{e^{-i \tilde{\omega}}}{\tilde{\omega}^{2-\alpha}} e^{\frac{1}{2} \pi i(2-\alpha)} \gamma(2-\alpha,-2 i \tilde{\omega})-Z_{0}^{(\alpha)}, \\
& Z_{2}^{(\alpha)}=\frac{2 e^{-i \tilde{\omega}}}{\tilde{\omega}^{3-\alpha}} e^{\frac{1}{2} \pi i(3-\alpha)} \gamma(3-\alpha,-2 i \tilde{\omega})-4 Z_{1}^{(\alpha)}-3 Z_{0}^{(\alpha)},
\end{aligned}
$$

where

$$
\gamma(a, z)=\int_{0}^{z} t^{a-1} e^{-t} d t, \quad \operatorname{Re}(a)>0
$$

is the incomplete gamma function [2, p. 260]. The recurrence relation (3.18) can be used to calculate $Z_{k}^{(\alpha)}$ stably in the forward direction provided $N \leq 2 \tilde{\omega}$. If $N>2 \tilde{\omega}$, we can compute the additional moments $Z_{k}^{(\alpha)}, 2 \tilde{\omega}<k \leq N$ stably by solving a boundary value 
problem with two starting values and one ending value [31]. To see this, let $n_{0}:=[2 \tilde{\omega}]$, where [ ] denotes the integer part, and choose a positive integer $N_{1} \geq \max \left\{n_{0}, N\right\}$. We then define a matrix $A=\left(a_{j, k}\right)_{j, k=1}^{N_{1}-n_{0}+1}$ of size $N_{1}-n_{0}+1$ by

$$
a_{j, k}= \begin{cases}i \tilde{\omega}\left(n_{0}+j-2\right), & k=j+1, \\ 2\left(n_{0}-\alpha+j\right)\left(n_{0}+j-2\right)+i \tilde{\omega}\left(n_{0}+j-3\right), & k=j, \\ 2\left(n_{0}+j-1\right)\left(n_{0}+\alpha+j-3\right)-i \tilde{\omega}\left(n_{0}+j\right), & k=j-1, \\ -i \tilde{\omega}\left(n_{0}+j-1\right), & k=j-2,\end{cases}
$$

and a vector $\mathbf{b}=\left(b_{1}, b_{2}, \ldots, b_{N_{1}-n_{0}+1}\right)^{T}$ by

$$
b_{j}= \begin{cases}-2^{2-\alpha} e^{i \tilde{\omega}}-\left(2 n_{0}\left(n_{0}+\alpha-2\right)-i \tilde{\omega}\left(n_{0}+1\right)\right) Z_{n_{0}-1}^{(\alpha)}+i \tilde{\omega} n_{0} Z_{n_{0}-2}^{(\alpha)}, & j=1, \\ -2^{2-\alpha} e^{i \tilde{\omega}}+i \tilde{\omega}\left(n_{0}+1\right) Z_{n_{0}-1}^{(\alpha)}, & j=2, \\ -2^{2-\alpha} e^{i \tilde{\omega}}, & j=3, \ldots, N_{1}-n_{0}, \\ -2^{2-\alpha} e^{i \tilde{\omega}}-i \tilde{\omega}\left(N_{1}-1\right) Z_{N_{1}+1}^{(\alpha)}, & j=N_{1}-n_{0}+1 .\end{cases}
$$

where the superscript ${ }^{T}$ stands for transpose. Here, the moments $Z_{n_{0}-2}^{(\alpha)}$ and $Z_{n_{0}-1}^{(\alpha)}$ can be obtained by using the forward recurrence (3.18), while the value of $Z_{N_{1}+1}^{(\alpha)}$ in the last component can be set equal to zero if the selected parameter $N_{1}$ is sufficiently large. We have that the vector consisting of the additional moments defined by

$$
\mathbf{Z}=\left(Z_{n_{0}}^{(\alpha)}, Z_{n_{0}+1}^{(\alpha)}, \ldots, Z_{N_{1}}^{(\alpha)}\right)^{T},
$$

is the unique solution of the linear system

$$
A \mathbf{x}=\mathbf{b} .
$$

To compute the integral on right hand side of (3.16), we note that

$$
\begin{aligned}
f_{-1}^{1}(y+1)^{-\alpha} \frac{e^{i \tilde{\omega} y}}{y-\tau} d y= & \frac{e^{i \tilde{\omega} \tau}}{(1+\tau)^{\alpha}}\left(i \pi+e^{-i \alpha \pi} \Gamma(1-\alpha) \Gamma(\alpha, i \tilde{\omega}(1+\tau))\right) \\
& -\frac{i e^{i \tilde{\omega}}}{\tilde{\omega}} \int_{0}^{\infty} e^{-t} \frac{1}{\left(2+\frac{i t}{\tilde{\omega}}\right)^{\alpha}\left(1-\tau+\frac{i t}{\tilde{\omega}}\right)} d t,
\end{aligned}
$$

where the last integral is free from singularity as $\tau \rightarrow-1$, and can therefore be conveniently evaluated by Gauss-Laguerre quadrature rule. For the case $\alpha=0$, it can be further written in a closed form (see [5]):

$$
\begin{aligned}
f_{-1}^{1} \frac{e^{i \tilde{\omega} y}}{y-\tau} d y= & \cos (\tilde{\omega} \tau)\left[\operatorname{Ci}\left(u_{1}\right)-\operatorname{Ci}\left(u_{2}\right)\right]-\sin (\tilde{\omega} \tau)\left[\operatorname{Si}\left(u_{1}\right)+\operatorname{Si}\left(u_{2}\right)\right] \\
& +i\left[\sin (\tilde{\omega} \tau)\left[\operatorname{Ci}\left(u_{1}\right)-\operatorname{Ci}\left(u_{2}\right)\right]+\cos (\tilde{\omega} \tau)\left[\operatorname{Si}\left(u_{1}\right)+\operatorname{Si}\left(u_{2}\right)\right]\right],
\end{aligned}
$$

where

$$
\operatorname{Ci}(x)=-\int_{x}^{\infty} \frac{\cos t}{t} d t, \quad x>0
$$


is the cosine integral, $u_{1}=\tilde{\omega}(1-\tau)$ and $u_{2}=\tilde{\omega}(1+\tau)$.

Based on the above discussion, we outline our algorithm with estimation of computational complexity as follows:

Algorithm I: Computation of $I_{1}(x)$

1. Choose a positive constant $a$ larger than $x$ and $N_{1}>\max \left\{n_{0}, N\right\}$.

2. Compute the coefficients $\left\{a_{k}^{N}\right\}_{k=0}^{N}$ in (3.11) by using FFT with $\mathcal{O}\left(N \log _{2} N\right)$ operations.

3. If $N \leq 2 \tilde{\omega}$, evaluate the moments $\left\{Z_{k}^{(\alpha)}\right\}_{k=0}^{N-1}$ from the recurrence relation (3.18) in $\mathcal{O}(N)$ operations. If $N>2 \tilde{\omega}$, we compute the additional moments $\left\{Z_{k}^{(\alpha)}\right\}_{k=n_{0}}^{N-1}$ by solving (3.22), in $\mathcal{O}\left(N_{1}\right)$ operation:

4. Compute $p_{N}(\tau)$ from its barycentric form in $\mathcal{O}(N)$ operations [4].

5. Calculate $Q_{N}^{(1)}(f, \tilde{\omega}, x)$ in $\mathcal{O}(N)$ operations by the Clenshaw algorithm:

$$
\begin{aligned}
& S_{-1}=0, S_{0}=\frac{1}{2} Z_{0}^{(\alpha)}, W_{0}=0, \\
& \left\{\begin{array}{l}
W_{k}=W_{k-1}+2 a_{k}^{N} S_{k-1}, \quad k=1,2, \ldots, N-1, \\
S_{k}=Z_{k}^{(\alpha)}+2 \tau S_{k-1}-S_{k-2},
\end{array}\right. \\
& Q_{N}^{(1)}(f, \tilde{\omega}, x)=\left(\frac{a}{2}\right)^{-\alpha} e^{i \tilde{\omega}}\left(W_{N-1}+S_{N-1} a_{N}^{N}+p_{N}(\tau) f_{-1}^{1}(y+1)^{-\alpha} \frac{e^{i \tilde{\omega} y}}{y-\tau} d y\right) .
\end{aligned}
$$

The integral $f_{-1}^{1}(y+1)^{-\alpha} \frac{e^{i \tilde{\omega} y}}{y-\tau} d y$ can be evaluated efficiently from (3.23) by using a Gauss-Laguerre quadrature rule.

The total computational complexity of computing $I_{1}(x)$ is $\mathcal{O}\left(N \log _{2} N\right)$ operations if $N \leq 2 \tilde{\omega}$ and is $\mathcal{O}\left(N \log _{2} N\right)+\mathcal{O}\left(N_{1}\right)$ operations if $N>2 \tilde{\omega}$. Therefore, even when we choose $N_{1}=\mathcal{O}\left(N \log _{2} N\right)$, the total computational complexity of computing $I_{1}(x)$ is still $\mathcal{O}\left(N \log _{2} N\right)$. Meanwhile, this cost is independent of $\omega$.

In the case $\alpha=0$, i.e., $f$ is analytic in the neighborhood of the origin, the above algorithm can be further simplified. Indeed, if $\alpha=0$, we have

$$
Z_{k}^{(0)}=\frac{1}{i \tilde{\omega}}\left(e^{i \tilde{\omega}}-(-1)^{k} e^{-i \tilde{\omega}}\right)-\frac{k}{i \tilde{\omega}} M_{k-1}, \quad k \geq 1 .
$$

Since $M_{k}$ satisfies a three-term recurrence relation (see [8, 33])

$$
M_{l}+\frac{2 l}{i \tilde{\omega}} M_{l-1}-M_{l-2}=\frac{2}{i \tilde{\omega}}\left(e^{i \tilde{\omega}}-(-1)^{l} e^{-i \tilde{\omega}}\right), \quad l \geq 2,
$$

\footnotetext{
†This can be done with a minor adaptation of Oliver's method for the LU decomposition of a tridiagonal matrix [27]. We omit the details.
} 
with initial values $M_{0}=\frac{2 \sin \tilde{\omega}}{\tilde{\omega}}, M_{1}=4 i\left(\frac{\sin \tilde{\omega}}{\tilde{\omega}^{2}}-\frac{\cos \tilde{\omega}}{\tilde{\omega}}\right)$, the four-term recurrence relation (3.18) can readily be reduced to a three-term recurrence relation. In practice, one can evaluate $M_{k}$ from (3.27) for $N \leq \tilde{\omega}$. If $N>\tilde{\omega}$, the additional moments $M_{k}, \tilde{\omega}<k \leq N$ can be evaluated stably by solving a tridiagonal system [8]. We then have the following simpler algorithm:

Algorithm II: Computation of $I_{1}(x)$ with $\alpha=0$

1. Choose a positive constant $a$ larger than $x$.

2. Compute the coefficients $\left\{a_{k}^{N}\right\}_{k=0}^{N}$ by using FFT in $\mathcal{O}\left(N \log _{2} N\right)$ operations.

3. Evaluate the moments $\left\{M_{k}\right\}_{k=0}^{N-1}$ by (3.27) if $N \leq \tilde{\omega}$. If $N>\tilde{\omega}$, we compute the additional moments $\left\{M_{k}\right\}_{k=[\widetilde{\omega}]}^{N-1}$ by the second phase algorithm in [8]. Then we evaluate $\left\{Z_{k}^{(0)}\right\}_{k=0}^{N-1}$ from (3.26), in $\mathcal{O}(N)$ operations.

4. Compute $p_{N}(\tau)$ from its barycentric form in $\mathcal{O}(N)$ operations [4].

5. Calculate $Q_{N}^{(1)}(f, \tilde{\omega}, x)$ in $\mathcal{O}(N)$ operations by the Clenshaw algorithm:

$$
\begin{aligned}
& S_{-1}=0, S_{0}=\frac{1}{2} Z_{0}^{(0)}, W_{0}=0, \\
& \left\{\begin{array}{l}
W_{k}=W_{k-1}+2 a_{k}^{N} S_{k-1}, \quad k=1,2, \ldots, N-1, \\
S_{k}=Z_{k}^{(0)}+2 \tau S_{k-1}-S_{k-2},
\end{array}\right. \\
& Q_{N}^{(1)}(f, \tilde{\omega}, x)=e^{i \tilde{\omega}}\left(W_{N-1}+S_{N-1} a_{N}^{N}+p_{N}(\tau) f_{-1}^{1} \frac{e^{i \tilde{\omega} y}}{y-\tau} d y\right) .
\end{aligned}
$$

The integral $f_{-1}^{1} \frac{e^{i \tilde{\omega} y}}{y-\tau} d y$ can be evaluated from (3.24).

The computational complexity is $\mathcal{O}\left(N \log _{2} N\right)$ operations.

\subsubsection{Computation of $I_{2}(x)$}

The computation of $I_{2}(x)$ is relatively easy, since the integrand does not have singularity. Under the same assumptions as in Lemma 2.1, it is readily seen that

$$
I_{2}(x)=\int_{a}^{\infty} \frac{f(t)}{t-x} e^{i \omega t} d t=i e^{i \omega a} \int_{0}^{\infty} \frac{f(a+i p)}{a-x+i p} e^{-\omega p} d p .
$$

As $a$ is an arbitrary real number larger than $x$, we may select it so that the integrand of the last integral in (3.28) is well behaved. Thus, this integral can be computed efficiently by Gauss-Laguerre quadrature rule:

$$
I_{2}(x) \simeq Q_{n}^{(2)}(f, \omega, x)=\frac{i e^{i \omega a}}{\omega} \sum_{k=1}^{n} w_{k} \frac{f\left(a+\frac{i t_{k}}{\omega}\right)}{a-x+\frac{i t_{k}}{\omega}},
$$

where $\left\{t_{k}, w_{k}\right\}$ are the nodes and weights of the Gauss-Laguerre quadrature associated with the weight $e^{-t}$. 
Example 3.4. We apply quadrature rule $Q_{n}^{(2)}(f, \omega, x)$ in (3.29) to calculate $I_{2}(x)$ with $f(t)=\sqrt{t} /(1+t)$ and $x=0.02$. The error is presented in Figure 5. We can see that more accurate approximations are obtained as $a$ increases. For each fixed $a$, more accurate approximations are obtained as $\omega$ increases.
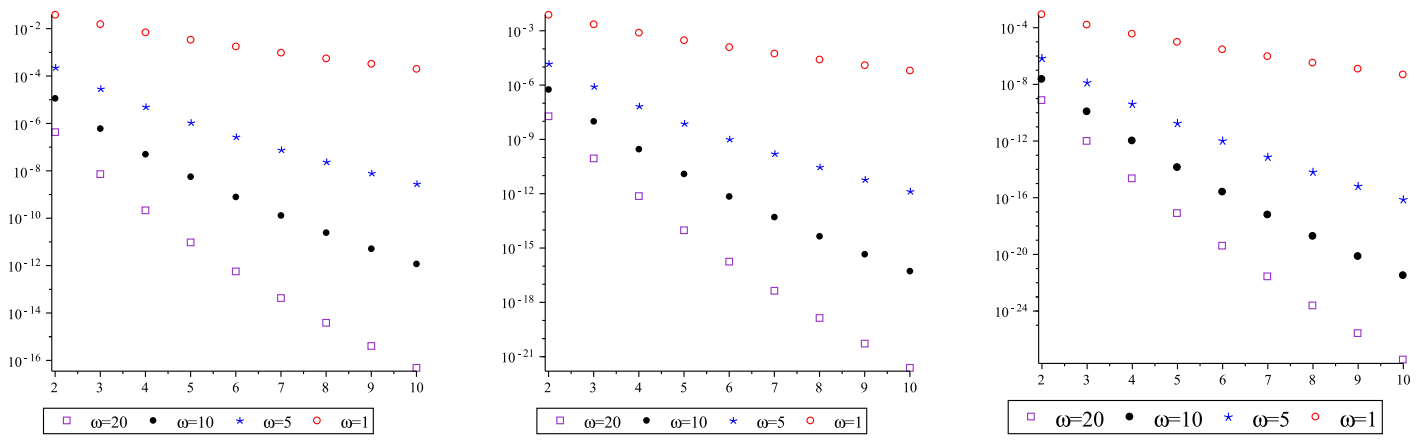

Figure 5: The error of the quadrature $Q_{n}^{(2)}(f, \omega, x)$ for $a=1$ (left), $a=2$ (middle) and $a=4$ (right) with $f(t)=\sqrt{t} /(1+t)$ and $n$ ranging from 2 to 10 . Here we choose $x=0.02$.

\subsubsection{Numerical examples}

We present in this section some numerical experiments to illustrate the efficiency of numerical methods presented in the above two sections.

Example 3.5. Let us consider the integral (2.33) with $c=1$ and $x$ close to zero. The exact solution is given in (3.4). Since $\alpha=0$ in this case, we use Algorithm II to compute $I_{1}(x)$, and the Gauss-Laguerre quadrature rule $Q_{n}^{(2)}(f, \omega, x)$ defined in (3.29) to compute $I_{2}(x)$. The absolute error for several values of $x$ is presented in Table 1, which indicates the proposed method is uniformly accurate as $x \rightarrow 0$. The absolute error for several values of $\omega$ with $a=1$ and $x=0.02$ is presented in Table 2 . We can see that the convergence is quite rapid as $N$ and $n$ increase.

Example 3.6. We consider (2.40) with $x$ close to the origin. Since $\alpha=1 / 2$ in this case, we use Algorithm I to compute $I_{1}(x)$ and the Gauss-Laguerre quadrature rule $Q_{n}^{(2)}(f, \omega, x)$ in (3.29) to compute $I_{2}(x)$. For simplicity, we choose $N_{1}=2 N$ when $N>2 \widetilde{\omega}$ in our implementation and the last integral in (3.23) is computed by using $32-$ point Gauss-Laguerre quadrature. The absolute error is presented in Table 3 for $a=1$ and $\omega=10$, which implies that the convergence is quite rapid as $N$ and $n$ increase. In Table 4 we present the absolute error for several values of $\omega$ with fixed $a$ and $x$. As we can see, the accuracy greatly improves as $\omega$ increases.

Remark 3.7. When $\alpha=0$, the uniform convergence of the quadrature rule (3.13) has been proved in [33]. When $0<\alpha<1$, however, we don't currently know whether the uniform convergence still holds. Numerical results show that the quadrature rule (3.13) remains accurate when $x$ is small, as can be observed from Table 3 . 
Table 1: Absolute error in computing (2.33) with $c=1, a=1, \omega=10$ and $x=10^{-\delta}$.

\begin{tabular}{ccllll}
\hline$n$ & $N$ & $\delta=1$ & $\delta=2$ & $\delta=3$ & $\delta=4$ \\
\hline 4 & 4 & $1.22 \times 10^{-5}$ & $3.80 \times 10^{-5}$ & $3.52 \times 10^{-5}$ & $3.42 \times 10^{-5}$ \\
& 8 & $3.30 \times 10^{-7}$ & $1.83 \times 10^{-7}$ & $1.73 \times 10^{-7}$ & $1.72 \times 10^{-7}$ \\
& 16 & $3.30 \times 10^{-7}$ & $1.83 \times 10^{-7}$ & $1.73 \times 10^{-7}$ & $1.72 \times 10^{-7}$ \\
8 & 4 & $1.19 \times 10^{-5}$ & $3.80 \times 10^{-5}$ & $3.52 \times 10^{-5}$ & $3.41 \times 10^{-5}$ \\
& 8 & $2.69 \times 10^{-10}$ & $1.09 \times 10^{-10}$ & $1.04 \times 10^{-10}$ & $1.03 \times 10^{-10}$ \\
& 16 & $2.86 \times 10^{-10}$ & $1.09 \times 10^{-10}$ & $9.96 \times 10^{-11}$ & $9.87 \times 10^{-11}$ \\
16 & 4 & $1.19 \times 10^{-5}$ & $3.80 \times 10^{-5}$ & $3.52 \times 10^{-5}$ & $3.41 \times 10^{-5}$ \\
& 8 & $2.37 \times 10^{-11}$ & $2.65 \times 10^{-12}$ & $9.36 \times 10^{-12}$ & $8.00 \times 10^{-12}$ \\
& 16 & $1.30 \times 10^{-14}$ & $2.97 \times 10^{-15}$ & $2.58 \times 10^{-15}$ & $2.54 \times 10^{-15}$ \\
\hline
\end{tabular}

Table 2: Absolute error in computing (2.33) with $c=1, a=1, x=0.02$ and several values of $\omega$.

\begin{tabular}{ccllll}
\hline$n$ & $N$ & $\omega=5$ & $\omega=20$ & $\omega=80$ & $\omega=320$ \\
\hline 4 & 4 & $2.84 \times 10^{-5}$ & $2.30 \times 10^{-5}$ & $1.56 \times 10^{-5}$ & $1.73 \times 10^{-5}$ \\
& 8 & $1.81 \times 10^{-5}$ & $8.92 \times 10^{-10}$ & $1.28 \times 10^{-11}$ & $1.81 \times 10^{-11}$ \\
& 16 & $1.81 \times 10^{-5}$ & $8.69 \times 10^{-10}$ & $4.89 \times 10^{-15}$ & $1.92 \times 10^{-20}$ \\
8 & 4 & $1.65 \times 10^{-5}$ & $2.30 \times 10^{-5}$ & $1.56 \times 10^{-5}$ & $1.73 \times 10^{-5}$ \\
& 8 & $8.16 \times 10^{-10}$ & $3.12 \times 10^{-11}$ & $1.28 \times 10^{-11}$ & $1.81 \times 10^{-11}$ \\
& 16 & $1.08 \times 10^{-10}$ & $2.00 \times 10^{-14}$ & $8.08 \times 10^{-24}$ & $3.65 \times 10^{-25}$ \\
16 & 4 & $1.66 \times 10^{-5}$ & $2.30 \times 10^{-5}$ & $1.56 \times 10^{-5}$ & $1.73 \times 10^{-5}$ \\
& 8 & $8.69 \times 10^{-11}$ & $3.12 \times 10^{-11}$ & $1.28 \times 10^{-11}$ & $1.81 \times 10^{-11}$ \\
& 16 & $7.49 \times 10^{-11}$ & $5.44 \times 10^{-21}$ & $2.78 \times 10^{-25}$ & $3.65 \times 10^{-25}$ \\
\hline
\end{tabular}

\subsection{The regime $x=0$}

When $x=0$, we need to deal with the Hadamard finite-part integral $f_{0}^{\infty} e^{i \omega t} \frac{f(t)}{t} d t$, which is introduced in Section 2.2. In view of (2.27), it suffices to find a fast method for evaluating the integral

$$
\int_{0}^{\infty} \frac{f(t)-a_{0} t^{-\alpha}}{t} e^{i \omega t} d t
$$

or equivalently,

$$
\int_{0}^{\infty} t^{-\alpha} g(t) e^{i \omega t} d t
$$

where

$$
g(t)=t^{\alpha} \frac{f(t)-a_{0} t^{-\alpha}}{t}
$$


Table 3: Absolute error in computing (2.40) with $a=1, \omega=10$ and $x=10^{-\delta}$.

\begin{tabular}{ccllll}
\hline$n$ & $N$ & $\delta=1$ & $\delta=2$ & $\delta=3$ & $\delta=4$ \\
\hline 4 & 4 & $1.53 \times 10^{-3}$ & $3.56 \times 10^{-3}$ & $4.56 \times 10^{-3}$ & $4.67 \times 10^{-3}$ \\
& 8 & $1.31 \times 10^{-6}$ & $1.63 \times 10^{-7}$ & $2.87 \times 10^{-6}$ & $3.25 \times 10^{-6}$ \\
& 16 & $1.16 \times 10^{-7}$ & $4.51 \times 10^{-8}$ & $4.08 \times 10^{-8}$ & $4.03 \times 10^{-8}$ \\
8 & 4 & $1.53 \times 10^{-3}$ & $3.56 \times 10^{-3}$ & $4.56 \times 10^{-3}$ & $4.67 \times 10^{-3}$ \\
& 8 & $1.23 \times 10^{-6}$ & $1.29 \times 10^{-7}$ & $2.89 \times 10^{-6}$ & $3.27 \times 10^{-6}$ \\
& 16 & $9.20 \times 10^{-11}$ & $2.22 \times 10^{-11}$ & $1.62 \times 10^{-11}$ & $1.51 \times 10^{-11}$ \\
16 & 4 & $1.53 \times 10^{-3}$ & $3.56 \times 10^{-3}$ & $4.56 \times 10^{-3}$ & $4.67 \times 10^{-3}$ \\
& 8 & $1.22 \times 10^{-6}$ & $1.29 \times 10^{-7}$ & $2.89 \times 10^{-6}$ & $3.27 \times 10^{-6}$ \\
& 16 & $1.39 \times 10^{-12}$ & $2.46 \times 10^{-12}$ & $1.33 \times 10^{-12}$ & $2.38 \times 10^{-12}$ \\
\hline
\end{tabular}

Table 4: Absolute error in computing (2.40) with $a=1, x=0.02$ and several values of $\omega$

\begin{tabular}{ccllll}
\hline$n$ & $N$ & $\omega=5$ & $\omega=20$ & $\omega=80$ & $\omega=320$ \\
\hline 4 & 8 & $5.50 \times 10^{-6}$ & $2.02 \times 10^{-6}$ & $2.04 \times 10^{-6}$ & $2.38 \times 10^{-6}$ \\
& 16 & $5.08 \times 10^{-6}$ & $2.15 \times 10^{-10}$ & $1.23 \times 10^{-12}$ & $2.60 \times 10^{-12}$ \\
& 32 & $5.08 \times 10^{-6}$ & $2.15 \times 10^{-10}$ & $1.19 \times 10^{-15}$ & $4.65 \times 10^{-21}$ \\
8 & 8 & $2.06 \times 10^{-6}$ & $2.02 \times 10^{-6}$ & $2.04 \times 10^{-6}$ & $2.38 \times 10^{-6}$ \\
& 16 & $2.41 \times 10^{-8}$ & $4.17 \times 10^{-13}$ & $1.23 \times 10^{-12}$ & $2.60 \times 10^{-12}$ \\
& 32 & $2.41 \times 10^{-8}$ & $3.86 \times 10^{-15}$ & $2.59 \times 10^{-24}$ & $6.75 \times 10^{-25}$ \\
16 & 8 & $2.08 \times 10^{-6}$ & $2.02 \times 10^{-6}$ & $2.04 \times 10^{-6}$ & $2.38 \times 10^{-6}$ \\
& 16 & $1.37 \times 10^{-11}$ & $4.13 \times 10^{-13}$ & $1.23 \times 10^{-12}$ & $2.60 \times 10^{-12}$ \\
& 32 & $1.39 \times 10^{-11}$ & $9.01 \times 10^{-22}$ & $1.11 \times 10^{-24}$ & $6.75 \times 10^{-25}$ \\
\hline
\end{tabular}

Since $g(t)$ is holomorphic in the first quadrant, which follows from the assumptions in Lemma 2.1 and Theorem 2.2, we can apply Gaussian quadrature rules as proposed by Wong [36]:

$$
\int_{0}^{\infty} t^{-\alpha} g(t) e^{i \omega t} d t=\frac{\exp \left((1-\alpha) \frac{i \pi}{2}\right)}{\omega^{1-\alpha}} \sum_{k=1}^{n} w_{k} g\left(\frac{i t_{k}}{\omega}\right)+E_{n}(g),
$$

where $\left\{t_{k}, w_{j}\right\}_{j=1}^{n}$ are the nodes and weights of the generalized Gauss-Laguerre quadrature with respect to weight $t^{-\alpha} e^{-t}$. The reminder $E_{n}(g)$ is given by

$$
E_{n}(g)=\frac{n ! \Gamma(n-\alpha+1)}{(2 n) ! \omega^{2 n-\alpha+1}} e^{(2 n-\alpha+1) \frac{i \pi}{2}} g^{(2 n)}(i \xi / \omega), \quad 0<\xi<\infty .
$$


Therefore, we can approximate $f_{0}^{\infty} e^{i \omega t} \frac{f(t)}{t} d t$ by

$$
\begin{cases}\frac{e^{\frac{\pi}{2}(2-\alpha) i} \omega^{\alpha}}{\alpha} \Gamma(1-\alpha) a_{0}+\frac{\exp \left((1-\alpha) \frac{i \pi}{2}\right)}{\omega^{1-\alpha}} \sum_{k=1}^{n} w_{k} g\left(\frac{i t_{k}}{\omega}\right), & \text { if } 0<\alpha<1, \\ \left(i \frac{\pi}{2}-\gamma-\log \omega\right) f(0)+\sum_{k=1}^{n} w_{k} \frac{f\left(\frac{i t_{k}}{\omega}\right)-f(0)}{t_{k}}, & \text { if } \alpha=0,\end{cases}
$$

Example 3.8. We use quadrature rule (3.34) to approximate (2.27) with $f(t)=e^{-t}$ and $f(t)=\sqrt{t} /(1+t)$, which corresponds to $\alpha=0$ and $\alpha=\frac{1}{2}$ respectively, for several values of $\omega$. The absolute errors are shown in Figure 6. Clearly, the convergence of the quadrature rule (3.34) is rapid and satisfactory.
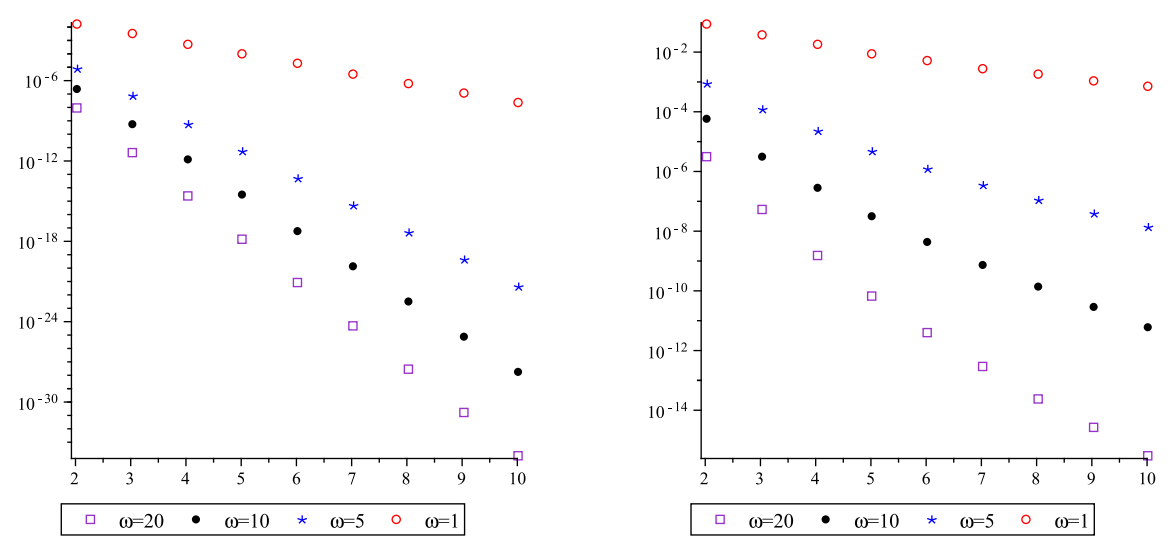

Figure 6: The error of the quadrature (3.34) for $f(t)=e^{-t}$ (left) and $f(t)=\sqrt{t} /(1+t)$ (right) with $n$ ranging from 2 to 10.

\section{Extensions}

Oscillatory Hilbert transforms with Bessel type oscillators are defined by

$$
H^{+}\left(f(t) J_{\nu}(\omega t)\right)(x):=f_{0}^{\infty} \frac{f(t)}{t-x} J_{\nu}(\omega t) d t
$$

and

$$
H^{+}\left(f(t) Y_{\nu}(\omega t)\right)(x):=f_{0}^{\infty} \frac{f(t)}{t-x} Y_{\nu}(\omega t) d t,
$$

where $J_{\nu}(t)$ and $Y_{\nu}(t)$ are the Bessel functions of the first and second kind, respectively. Such kind of transformations have applications in physics such as water-wave radiation problem [24. It turns out that Lemma 2.1 can also be extended to oscillatory Bessel Hilbert transforms, by using similar ideas. 
Lemma 4.1. Suppose that $f$ has an analytic continuation to the right half plane, except possibly a branch point at the origin, and there exist constants $M>0, \delta<\frac{3}{2}$ and $0 \leq d<\omega$ such that

$$
|f(z)| \leq M|z|^{\delta} e^{d \operatorname{Im}(z)},
$$

as $|z| \rightarrow \infty$ in the right-half plane. Then,

$$
H^{+}\left(f(t) J_{\nu}(\omega t)\right)(x)=-\pi f(x) Y_{\nu}(\omega x)-\frac{1}{\pi} \int_{0}^{\infty} \frac{K_{\nu}(\omega y)}{y^{2}+x^{2}} g_{1}(y) d y
$$

and

$$
H^{+}\left(f(t) Y_{\nu}(\omega t)\right)(x)=\pi f(x) J_{\nu}(\omega x)+\frac{i}{\pi} \int_{0}^{\infty} \frac{K_{\nu}(\omega y)}{y^{2}+x^{2}} g_{2}(y) d y
$$

whenever the integrals exist, where $K_{\nu}(t)$ is the modified Bessel function of the second kind and

$$
g_{j}(y)=(x+i y) e^{-\frac{\nu}{2} \pi i} f(i y)+(-1)^{j+1}(x-i y) e^{\frac{\nu}{2} \pi i} f(-i y), \quad j=1,2 .
$$

Proof. We only give a sketched proof of (4.2), since the proof of (4.3) can be handled in a similar manner. On account of the identity (see [2, Eq. 9.6.4])

$$
i \pi J_{\nu}(z)=e^{-\frac{\nu}{2} \pi i} K_{\nu}\left(z e^{-\frac{1}{2} \pi i}\right)-e^{\frac{\nu}{2} \pi i} K_{\nu}\left(z e^{\frac{1}{2} \pi i}\right), \quad|\arg z| \leq \frac{\pi}{2},
$$

it follows

$$
\begin{aligned}
& f_{0}^{\infty} \frac{f(t)}{t-x} J_{\nu}(\omega t) d t \\
& =\frac{1}{i \pi}\left[e^{-\frac{\nu}{2} \pi i} f_{0}^{\infty} \frac{f(t)}{t-x} K_{\nu}(-i \omega t) d t-e^{\frac{\nu}{2} \pi i} f_{0}^{\infty} \frac{f(t)}{t-x} K_{\nu}(i \omega t) d t\right] .
\end{aligned}
$$

For the first integral on the right hand side of (4.4), by considering the same contour as shown in Figure 1, we obtain from Cauchy's theorem that

$$
\begin{aligned}
& f_{0}^{R} \frac{f(t)}{t-x} K_{\nu}(-i \omega t) d t \\
& =i \pi f(x) K_{\nu}(-i \omega x)-\int_{\Gamma_{R}} \frac{f(z)}{z-x} K_{\nu}(-i \omega z) d z+i \int_{0}^{R} \frac{f(i y)}{i y-x} K_{\nu}(\omega y) d y .
\end{aligned}
$$

Recall the asymptotic expansion of the modified Bessel function $K_{\nu}(z)$ [2, p. 378]

$$
K_{\nu}(z) \sim \sqrt{\frac{\pi}{2 z}} e^{-z}\left[1+\frac{4 \nu^{2}-1}{8 z}+\mathcal{O}\left(z^{-2}\right)\right], \quad z \rightarrow \infty, \quad|\arg z|<\frac{3 \pi}{2} .
$$


We have the following estimation of the integral over $\Gamma_{R}$ for large $R$

$$
\begin{aligned}
\left|\int_{\Gamma} \frac{f(z)}{z-x} K_{\nu}(-i \omega z) d z\right| & =\left|\int_{0}^{\frac{\pi}{2}} \frac{f\left(R e^{i \theta}\right)}{R e^{i \theta}-x} K_{\nu}\left(-i \omega R e^{i \theta}\right) i R e^{i \theta} d \theta\right| \\
& \leq \frac{M R^{1+\delta}}{R-x} \int_{0}^{\frac{\pi}{2}} e^{d R \sin \theta}\left|K_{\nu}\left(-i \omega R e^{i \theta}\right)\right| d \theta \\
& \leq \frac{M R^{1+\delta}}{R-x} \sqrt{\frac{\pi}{2 \omega R}} \int_{0}^{\frac{\pi}{2}} e^{-(\omega-d) R \sin \theta}\left(1+\mathcal{O}\left(R^{-1}\right)\right) d \theta \\
& \rightarrow 0, \quad \text { as } R \rightarrow \infty .
\end{aligned}
$$

Hence, letting $R \rightarrow \infty$ in (4.5), we arrive at

$$
f_{0}^{\infty} \frac{f(t)}{t-x} K_{\nu}(-i \omega t) d t=i \pi f(x) K_{\nu}(-i \omega x)+i \int_{0}^{\infty} \frac{f(i y)}{i y-x} K_{\nu}(\omega y) d y
$$

Similarly, we can deform the integration path to the negative imaginary axis for the second integral on the right hand side of (4.4) and get

$$
f_{0}^{\infty} \frac{f(t)}{t-x} K_{\nu}(i \omega t) d t=-i \pi f(x) K_{\nu}(i \omega x)+i \int_{0}^{\infty} \frac{f(-i y)}{i y+x} K_{\nu}(\omega y) d y .
$$

A combination of (4.4), (4.6) and (4.7) gives us (4.2).

From the above lemma, we establish several interesting identities with $\nu=0,1$, which have not been found in classical reference books [2, 12] and which might have important applications in practice:

Corollary 4.2. We have

$$
f_{0}^{\infty} \frac{J_{\nu}(\omega t)}{t-x} d t=(-1)^{\nu+1} \frac{\pi}{2}\left[\mathbf{H}_{-\nu}(\omega x)+(-1)^{\nu} Y_{\nu}(\omega x)\right], \quad \nu=0,1
$$

and

$$
f_{0}^{\infty} \frac{Y_{0}(\omega t)}{t-x} d t=\pi J_{0}(\omega x)-\frac{2}{\pi} S_{-1,0}(\omega x)
$$

and

$$
f_{0}^{\infty} \frac{t Y_{1}(\omega t)}{t-x} d t=\pi x J_{1}(\omega x)-\frac{4 x}{\pi} S_{-2,1}(\omega x)
$$

where $\mathbf{H}_{\nu}(z)$ is the Struve function and $S_{\mu, \nu}(z)$ is the Lommel function of the second kind.

Proof. With $f=1$ and $\nu=0,1$ in (4.2), it follows that

$$
f_{0}^{\infty} \frac{J_{\nu}(\omega t)}{t-x} d t= \begin{cases}-\pi Y_{0}(\omega x)-\frac{2 x}{\pi} \int_{0}^{\infty} \frac{K_{0}(\omega y)}{y^{2}+x^{2}} d y, & \text { if } \nu=0 \\ -\pi Y_{1}(\omega x)-\frac{2}{\pi} \int_{0}^{\infty} \frac{y K_{1}(\omega y)}{y^{2}+x^{2}} d y, & \text { if } \nu=1 .\end{cases}
$$


Recall the identity (see [12, Eq. 6.566.3])

$\int_{0}^{\infty} \frac{y^{\nu} K_{\nu}(\omega y)}{y^{2}+x^{2}} d y=\frac{\pi^{2} x^{\nu-1}}{4 \cos (\nu \pi)}\left[\mathbf{H}_{-\nu}(\omega x)-Y_{-\nu}(\omega x)\right], \quad \omega>0, \quad \operatorname{Re} x>0, \quad \operatorname{Re} \nu>-\frac{1}{2}$.

Formula (4.8) then follows from inserting the above identity with $\nu=0$ and $\nu=1$, respectively.

To show (4.9), we set $f=1$ and $\nu=0$ in (4.3) and obtain

$$
f_{0}^{\infty} \frac{Y_{0}(\omega t)}{t-x} d t=\pi J_{0}(\omega x)-\frac{2}{\pi} \int_{0}^{\infty} \frac{y K_{0}(\omega y)}{y^{2}+x^{2}} d y
$$

Since

$$
\int_{0}^{\infty} y^{1+\nu}\left(y^{2}+x^{2}\right)^{\mu} K_{\nu}(\omega y) d y=2^{\nu} \Gamma(\nu+1) x^{\nu+\mu+1} \omega^{-1-\mu} S_{\mu-\nu, \mu+\nu+1}(\omega x)
$$

for $\operatorname{Re} x>0, \operatorname{Re} \omega>0$ and $\operatorname{Re} \nu>-1$ (see [12, Eq. 6.565.7]), substituting the above identity with $\nu=0$ and $\mu=-1$ into (4.12) gives us (4.9).

Finally, the proof of (4.10) is similar to that of (4.9), and we omit the details here.

We expect that Lemma 4.1 might play an important role in the asymptotic and numerical study of oscillatory Hilbert transforms. Note that the modified Bessel functions $K_{\nu}$ have a non-integrable singularity at 0 for $\nu \geq 1$.

Finally, we like to recall that the numerical method proposed in [3] generalized steepest descent-based methods for Fourier-type integrals of the form (1.1) to oscillatory transforms of the form

$$
\int_{0}^{\infty} f(x) H(\omega x) d x
$$

where $H$ can be, e.g., a Bessel function. We expect that this method can be extended in turn to compute oscillatory Hilbert transforms with more general oscillators as well.

\section{Concluding remarks}

In this paper, we have considered asymptotic expansions and fast computation of the oscillatory Hilbert transforms (1.4). Unlike previous work, which focused on the behaviour of oscillatory Hilbert transforms for large $x$, we derive asymptotic expansions of such transforms for large $\omega$. These expansions clarify the asymptotic behaviour for large $\omega$ and provide a powerful approach for designing efficient and accurate approximation methods.

Numerical methods for the calculation of the oscillatory Hilbert transforms are presented. We classify our discussion into three regimes, namely, $x=\mathcal{O}(1)$ or $x \gg 1$, $0<x \ll 1$ and $x=0$. For each regime, we have designed efficient numerical approaches. Even for small values of $\omega$, our proposed methods remain quite accurate. Numerical examples are provided to confirm our analysis. 
In the implementation of our methods, the function $f(x)$ is required to be analytic in the first quadrant of the complex plane. It seems that this requirement is more restrictive. If $f(x)$ is a differentiable but not analytic function, then we may construct a suitable Filon-type method which utilizes Hermite interpolation to compute such transforms. These results will be reported in our future work.

\section{Acknowledgement}

We thank Andreas Asheim for helpful discussions.

\section{References}

[1] M. J. Ablowitz and A. S. Fokas, Complex Variables: Introduction and Applications, Cambridge University Press, 2003.

[2] M. Abramowitz and I. A. Stegun, Handbook of Mathematical Functions, National Bureau of Standards, Washington, D.C., 1964.

[3] A. Asheim and D. Huybrechs, Complex Gaussian quadrature for oscillatory integral transforms, Report TW 594, 2011.

[4] J. P. Berrut and L. N. Trefethen, Barycentric Lagrange interpolation, SIAM Review, 46 (2004), 501-517.

[5] M. R. Capobianco, G. Criscuolo, On quadrature for Cauchy principal value integrals of oscillatory functions, J. Comput. Appl. Math., 156 (2003), 471-486.

[6] C. W. Clenshaw and A. R. Curtis, A method for numerical integration on an automatic computer, Numer. Math., 2 (1960), 197-205.

[7] A. Deaño and D. Huybrechs, Complex Gaussian quadrature of oscillatory integrals, Numer. Math., 112 (2009), 197-219.

[8] V. Domínguez, I. G. Graham and V. P. Smyshlyaev, Stability and error estimates for Filon-Clenshaw-Curtis rules for highly oscillatory integrals, IMA J. Numer. Anal., 31 (2011), 1253-1280.

[9] K. C. Chung, G. A. Evans and J. R. Webster, A method to generate generalized quadrature rules for oscillatory integrals, Appl. Numer. Math., 34 (2000), 85-93.

[10] P. J. Davis and P. Rabinowitz, Methods of Numerical Integration, Second Edition, Academic Press, 1984.

[11] W. M. Gentleman, Implementing Clenshaw-Curtis quadrature. II, Comm. ACM, 15 (1972), 343-346. 
[12] I. S. Gradshteyn, I. M. Ryzhik, Tables of Integrals, Series, and Products, 6th ed. San Diego, CA: Academic Press, 2000.

[13] T. Hasegawa and T. Torii, An automatic quadrature for Cauchy principal value integrals, Math. Comp., 56 (1991), 741-754.

[14] D. Huybrechs and S. Vandewalle, On the evaluation of highly oscillatory integrals by analytic continuation, SIAM J. Numer. Anal., 44 (2006), 1026-1048.

[15] A. Iserles and S. P. Nørsett, Efficient quadrature of highly oscillatory integrals using derivatives, Proc. Royal. Soc. A, 461 (2005), 1383-1399.

[16] A. Iserles and S. P. Nørsett, On quadrature methods for highly oscillatory integrals ans their implementation, BIT, 44 (2004), 755-772.

[17] F. W. King, Hilbert transforms: Volume 1, Cambridge University Press, 2009.

[18] F. W. King, G. J. Smethells, G. T. Helleloid and P. J. Pelzl, Numerical evaluation of Hilbert transforms for oscillatory functions: A convergence accelerator approach, Comput. Phys. Comm., 145 (2002), 256-266.

[19] A. R. Krommer and C. W. Ueberhuber, Computational Integration, SIAM, Philadelphia, 1998.

[20] D. Levin, Fast integration of rapidly oscillatory functions, J. Comput. Appl. Math., 67 (1996), 95-101.

[21] D. Levin, Procedures for computing one- and two-dimensional integrals of functions with rapid irregular oscillations, Math. Comp., 38 (1982), 531-538.

[22] J. N. Lyness, The Euler Maclaurin expansion for the Cauchy principal value integral, Numer. Math., 46 (1985), 611-622.

[23] G. V. Milovanović, Numerical calculation of integrals involving oscillatory and singular kernels and some applications of quadratures, Comput. Math. Appl., 36 (1998), 19-39.

[24] P. A. Martin, On the null-field equations for water-wave radiation problems, J. Fluid Mech., 113 (1981), 315-332.

[25] G. Monegato and J. N. Lyness, The Euler-Maclaurin expansion and finite-part integrals, Numer. Math., 81 (1998), 273-291.

[26] G. E. Okecha, Quadrature formulae for Cauchy principal value integrals of oscillatory kind, Math. Comp., 49 (1987), 259-268.

[27] J. Oliver, Relative error propagation in the recursive solution of linear recurrence relations, Numer. Math., 9 (1967), 323-340. 
[28] S. Olver, Computing the Hilbert transform and its inverse, Math. Comp., 80 (2011), 1745-1767.

[29] S. Olver, Moment-free numerical integration of highly oscillatory functions, IMA. J. Numer. Anal., 26 (2006), 213-227.

[30] S. Olver, GMRES for the differentiation operator, SIAM. J. Numer. Anal., 47 (2009), 3359-3373.

[31] R. Piessens and M. Branders, On the computation of Fourier transforms of singular functions, J. Comput. Appl. Math., 43 (1992), 159-169.

[32] F. Ursell, Integrals with a large parameter: Hilbert transforms, Math. Proc. Camb. Soc., 93 (1983), 141-149.

[33] H. Wang and S. Xiang, Uniform approximations to Cauchy principal value integrals of oscillatory functions, Appl. Math. Comp., 215 (2009), 1886-1894.

[34] H. Wang and S. Xiang, On the evaluation of Cauchy principal value integrals of oscillatory functions, J. Comput. Appl. Math., 234 (2010), 95-100.

[35] R. Wong, Asymptotic expansion of the Hilbert transform, SIAM J. Math. Anal., 11 (1980), 92-99.

[36] R. Wong, Quadrature formulas for oscillatory integral transforms, Numer. Math., 39 (1982), 351-360.

[37] R. Wong, Asymptotic Approximations of Integrals, SIAM, Philadelphia, 2001.

[38] S. Xiang, Efficient Filon-type methods for $\int_{a}^{b} f(x) e^{i \omega g(x)} d x$, Numer. Math., 105 (2007), 633-658.

[39] S. Xiang, X. Chen and H. Wang, Error bounds for approximation in Chebyshev points, Numer. Math., 116 (2010), 463-491. 